\title{
Atomistic Insight into Tetraalkylphosphonium-Bis(oxalato)borate Ionic Liquid/Water Mixtures. I. Local Microscopic Structure
}

\author{
Yong-Lei Wang, ${ }^{* \dagger}{ }^{\dagger}$ Sten Sarman, ${ }^{\dagger}$ Sergei Glavatskih, ${ }^{\dagger, \S}$ Oleg N. Antzutkin, ${ }^{\|, \perp}$ Mark W. Rutland, ${ }^{\#, \nabla}$ \\ and Aatto Laaksonen $*, \dagger, \bigcirc$ \\ ${ }^{\dagger}$ Department of Materials and Environmental Chemistry, Arrhenius Laboratory, Stockholm University, SE-106 91 Stockholm, Sweden
${ }^{\ddagger}$ System and Component Design, KTH Royal Institute of Technology, SE-100 44 Stockholm, Sweden
${ }^{\S}$ Mechanical Construction and Production, Ghent University, B-9000 Ghent, Belgium
${ }^{{ }^{\$}}$ Chemistry of Interfaces, Luleå University of Technology, SE-971 87 Luleå, Sweden
${ }^{\perp}$ Department of Physics, Warwick University, CV4 7AL Coventry, United Kingdom
${ }^{\#}$ Surface and Corrosion Science, KTH Royal Institute of Technology, SE-100 44 Stockholm, Sweden
${ }^{\nabla}$ Chemistry, Materials and Surfaces, SP Technical Research Institute of Sweden, SE-114 86 Stockholm, Sweden
OStellenbosch Institute of Advanced Study (STIAS), Wallenberg Research Centre, Stellenbosch University, Marais Street,
Stellenbosch 7600, South Africa
}

ABSTRACT: Atomistic simulations have been performed to investigate the microscopic structural organization of aqueous solutions of trihexyltetradecylphosphonium bis(oxalato)borate $\left(\left[\mathrm{P}_{6,6,6,14}\right][\mathrm{BOB}]\right)$ ionic liquid (IL). The evolution of the microscopic liquid structure and the local ionic organization of $\mathrm{IL} /$ water mixtures as a function of the water concentration is visualized and systematically analyzed via radial and spatial distribution functions, coordination numbers, hydrogen bond network, and water clustering analysis. The microscopic liquid structure in neat IL is characterized by a connected apolar network composed of the alkyl chains of $\left[\mathrm{P}_{6,6,6,14}\right]$ cations and isolated polar domains consisting of the central segments of $\left[\mathrm{P}_{6,6,6,14}\right]$ cations and $[\mathrm{BOB}]$ anions, and the corresponding local ionic environment is

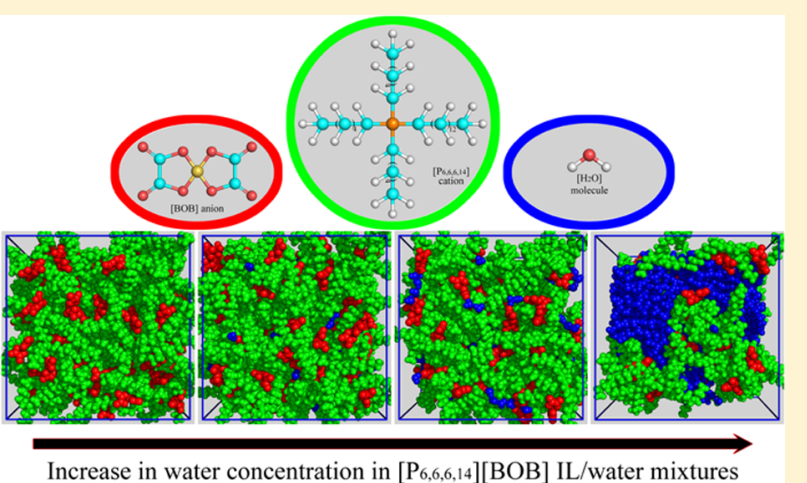
described by direct contact ion pairs. In IL/water mixtures with lower water mole fractions, the added water molecules are dispersed and embedded in cavities between neighboring ionic species and the local ionic structure is characterized by solvent-shared ion pairs through cation-water-anion triple complexes. With a gradual increase in the water concentration in IL/water mixtures, the added water molecules tend to aggregate and form small clusters, intermediate chain-like structures, large aggregates, and eventually a water network in water concentrated simulation systems. A further progressive dilution of $\mathrm{IL} /$ water mixtures leads to the formation of self-organized micelle-like aggregates characterized by a hydrophobic core and hydrophilic shell consisting of the central polar segments in $\left[\mathrm{P}_{6,6,6,14}\right]$ cations and $[\mathrm{BOB}]$ anions in a highly branched water network. The striking structural evolution of the $\left[\mathrm{P}_{6,6,6,14}\right][\mathrm{BOB}] \mathrm{IL} /$ water mixtures is rationalized by the competition between favorable hydrogen bonded interactions and strong electrostatic interactions between the polar segments in ionic species and the dispersion interactions between the hydrophobic alkyl chains in $\left[\mathrm{P}_{6,6,6,14}\right]$ cations.

\section{INTRODUCTION}

Ionic liquids (ILs) are special categories of molten salts with melting points below $100{ }^{\circ} \mathrm{C}$, which are usually composed of inorganic or organic anions and, most commonly, organic cations with typical bulky and asymmetric structures. ${ }^{1,2} \mathrm{~A}$ variety of ILs are liquids at room temperature, partially due to asymmetric ionic structures between conformationally flexible organic cations and anions, increasing the entropy and thus preventing crystallization. ${ }^{3}$ ILs have attracted significant and widespread attention in academic and industrial communities over the last two decades due to their unique properties, such as negligible volatility, exceptional thermal and electrochemical stabilities, wide electrochemical window, and excellent solvation capacity for both polar and apolar compounds. ${ }^{4,5}$ These desirable properties have resulted in their extensive investigations as functional materials in many potentially promising applications in diverse scientific and technological research areas, including materials synthesis and catalysis, ${ }^{6-8}$ lubrication and micronanotribology, ${ }^{9-14}$ gas separation (as solvents and

Received: January 22, 2015

Revised: $\quad$ March 25, 2015

Published: March 31, 2015 
membrane transport media), ${ }^{15,16}$ cellulose processing, ${ }^{17-19}$ and active pharmaceutical ingredients. ${ }^{20}$

Besides the extensively studied nitrogen-based ILs, such as imidazolium-, piperidinium-, pyrrolidinium-, pyridinium-, cholinium-, and guanidinium-based ILs, ${ }^{19,21,22}$ new IL families including sulfonium ${ }^{23-25}$ and phosphonium cations ${ }^{26}$ have attracted increasing interest in recent years. Phosphoniumbased ILs, many of which are composed of volume-occupying alkyl chains, ${ }^{27-29}$ present additional advantages compared with nitrogen-based ILs in large-scale industrial operations. The ILs consisting of tetraalkylphosphonium cations and chelated orthoborate anions exhibit outstanding tribological properties in steel-aluminum contacts, and are attractive as alternative high-performance lubricants compared with fully formulated engine oils. ${ }^{11,30}$ Therefore, the number of fundamental studies and industrial applications of phosphonium- and boron-based ILs is growing rapidly (for recent reviews, see refs 4, 9, 12, and 30-34 and references therein).

One of the most important issues in IL-related research and applications is the potential presence of impurities in IL samples. ${ }^{35,36}$ As an omnipresent compound, water is one of the most common contaminators found in ILs, on one hand due to the intrinsic hygroscopic nature of some $\mathrm{ILs}^{11,37}$ and on the other hand because many chemical processes (synthesis, extraction, etc.) involve water. ${ }^{15,17,18}$ It has been well documented that even a small amount of water can drastically alter physicochemical properties of ILs, which may lead to significant modifications in, for example, the rate and selectivity of chemical reactions taking place in their midst. ${ }^{38,39}$ As described in our previous work, the water content in freshly synthesized $\left[\mathrm{P}_{6,6,6,14}\right][\mathrm{BOB}]$ IL samples is $2.3-2.5 \mathrm{wt} \%,{ }^{11}$ and the corresponding water mole fraction is quite close to 0.50 (as shown in Table 1). This amount of water is difficult to remove

Table 1. Chemical Compositions of All Studied $\left[\mathbf{P}_{6,6,6,14}\right][\mathrm{BOB}] \mathrm{IL} /$ Water Mixtures with Varied Water Mole Fractions $^{a}$

$\begin{array}{ll}x_{\text {water }} & N \\ 0.0 & 96 \\ 0.10 & 96 \\ 0.20 & 96 \\ 0.33 & 96 \\ 0.50 & 96 \\ 0.67 & 88 \\ 0.75 & 8 \\ 0.80 & 72 \\ 0.90 & 64 \\ 0.95 & 48\end{array}$

$\begin{array}{rr}N_{\text {IL }} & N_{\text {wate }} \\ 96 & 0 \\ 96 & 11 \\ 96 & 24 \\ 96 & 48 \\ 96 & 96 \\ 88 & 176 \\ 80 & 240 \\ 72 & 288 \\ 64 & 576 \\ 48 & 912\end{array}$

$\begin{array}{rl}N_{\text {water }} & \text { wt }_{\text {water }} \\ 0 & 0.0 \\ 11 & 0.0031 \\ 24 & 0.0067 \\ 48 & 0.0132 \\ 96 & 0.0262 \\ 176 & 0.0510 \\ 240 & 0.0746 \\ 288 & 0.0970 \\ 576 & 0.1947 \\ 912 & 0.3379\end{array}$

${ }^{a} N_{\text {IL }}$ and $N_{\text {water }}$ are the numbers of $\left[\mathrm{P}_{6,6,6,14}\right][\mathrm{BOB}]$ ion pairs and water molecules in the studied simulation systems, respectively. The symbols $x_{\text {water }}$ and $\mathrm{wt}_{\mathrm{water}}$ denote the water mole and mass fractions in the $\left[\mathrm{P}_{6,6,6,14}\right][\mathrm{BOB}] \mathrm{IL} /$ water mixtures, respectively.

even after several iterative purifications including vacuum drying for a few hours at $85-90{ }^{\circ} \mathrm{C}$. The intrusion of water molecules may disturb the microscopic ionic structure, leading to the significant changes in liquid densities, and may also contribute to the non-negligible discrepancy of ionic group diffusion between experimental measurements and simulation results. ${ }^{11,40}$ Therefore, it is important to gain a molecular level understanding of $\left[\mathrm{P}_{6,6,6,14}\right][\mathrm{BOB}] \mathrm{IL} /$ water mixtures, not only because water is inevitably present in many practical applications but also because it provides a new opportunity to fine-tune the physicochemical properties of $\left[\mathrm{P}_{6,6,6,14}\right][\mathrm{BOB}]$ IL by adding small molecules, like water.

In this work, extended atomistic molecular dynamics simulations are performed to obtain a deeper insight into the microscopic liquid structure of $\left[\mathrm{P}_{6,6,6,14}\right][\mathrm{BOB}] \mathrm{IL} /$ water mixtures as a function of the water concentration. The evolution of the microscopic liquid structure and the local ionic organization of IL/water mixtures, characterized by radial and spatial distribution functions, coordination numbers, hydrogen bond network, as well as water clustering analysis, is investigated and discussed in detail. Four structural regimes are identified within the full water concentration range, in which the microscopic ionic environment is distinct and characterized by a structural heterogeneity. Such an ionic structural heterogeneity of $\left[\mathrm{P}_{6,6,6,14}\right][\mathrm{BOB}] \mathrm{IL} /$ water mixtures contributes directly to the striking thermodynamic quantities and particular transport properties, which will be systematically discussed in the following parts of this work.

\section{MODELS AND SIMULATION METHODOLOGY}

Atomistic Force Field. Schematic molecular structures and representative atom types in the $\left[\mathrm{P}_{6,6,6,14}\right]$ cation, the $[\mathrm{BOB}]$ anion, and the SPC/E water molecule are presented in Figure 1. The interaction parameters of atomistic $\left[\mathrm{P}_{6,6,6,14}\right]$ cation and [BOB] anion models stem from a systematically developed force field in our previous work, ${ }^{40}$ in which both inter- and intramolecular interaction parameters were first refined to achieve a quantitative description of the intermolecular ionic structure obtained from quantum chemistry $a b$ initio calculations, and thereafter tuned to fit vibration frequency data obtained from experimental measurements, ${ }^{11}$ respectively. The flexible SPC/E water model is employed in the present work, which can provide a good description of the structural and dynamic properties of bulk liquid water. ${ }^{41}$ The cross interaction parameters between unlike atoms are obtained by the LorentzBerthelot combining rule.

Simulation Methodology. Atomistic simulations of the $\left[\mathrm{P}_{6,6,6,14}\right][\mathrm{BOB}] \mathrm{IL} /$ water mixtures with varied water mole fractions ranging from 0.0 (neat IL) to 0.95 were performed in the present work. The numbers of $\left[\mathrm{P}_{6,6,6,14}\right][\mathrm{BOB}]$ ion pairs and water molecules in each simulation system depend on the IL/water mixtures and the computational feasibility. The detailed chemical compositions of all studied $\mathrm{IL} /$ water mixtures are listed in Table 1.

Atomistic molecular dynamics simulations were performed using the M.Dynamix package ${ }^{42}$ with standard three-dimensional periodic boundary conditions. The equations of motion were integrated using the Tuckerman-Berne double time step algorithm ${ }^{43}$ with short and long time steps of 0.2 and $2.0 \mathrm{fs}$, respectively. The short time step was used for integrating fast intramolecular vibrations and nonbonded interactions within $0.5 \mathrm{~nm}$, while the long time step was used for integrating van der Waals and electrostatic interactions, as well as dihedral angle motions, within a cutoff distance of $1.5 \mathrm{~nm}$. The electrostatic interactions between atom-centered point charges were treated with the standard Ewald summation method. All simulation systems were equilibrated for $8 \mathrm{~ns}$ in the isothermal-isobaric ensemble maintained using the NoséHoover thermostat at $333 \mathrm{~K}$ and barostat under 1 atm with time coupling constants of 1000 and $30 \mathrm{fs,} \mathrm{respectively.}$ Another 12 ns, canonical ensemble simulations were further 


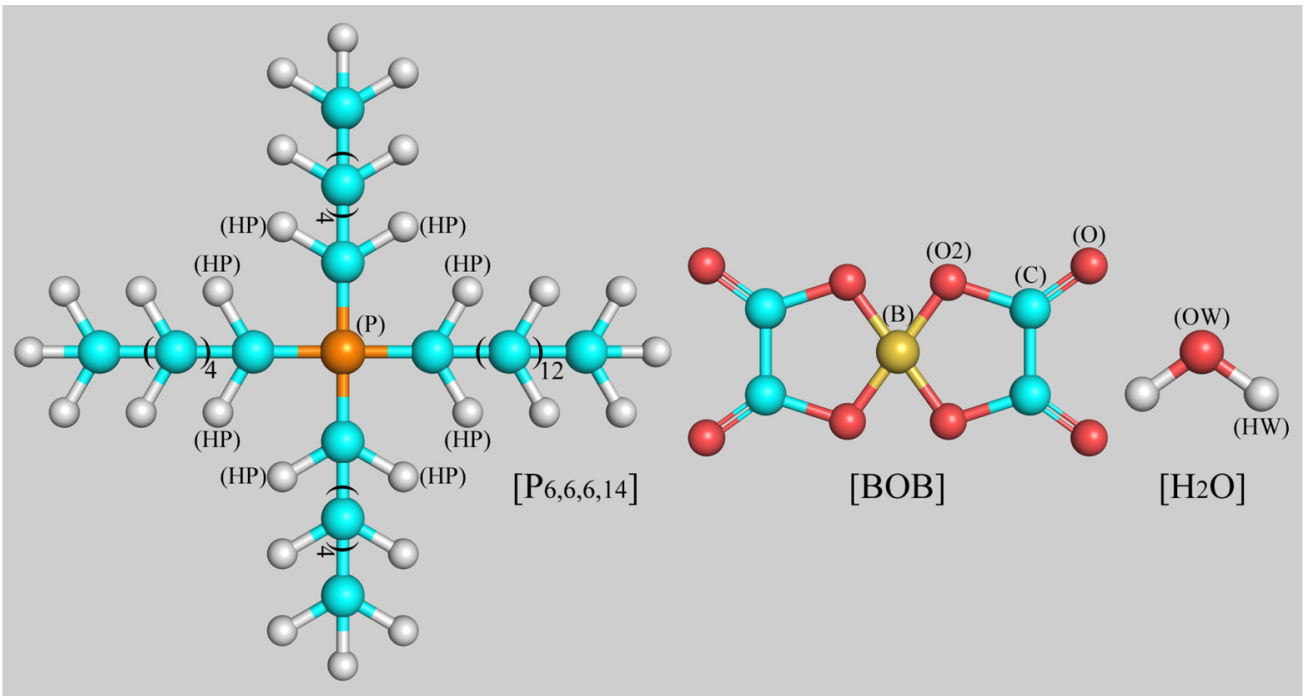

Figure 1. Molecular structures of the $\left[\mathrm{P}_{6,6,6,14}\right]$ cation, the $[\mathrm{BOB}]$ anion, and the SPC/E water molecule. Representative atom type notations of these three molecules are presented according to their symmetric atomic structures.
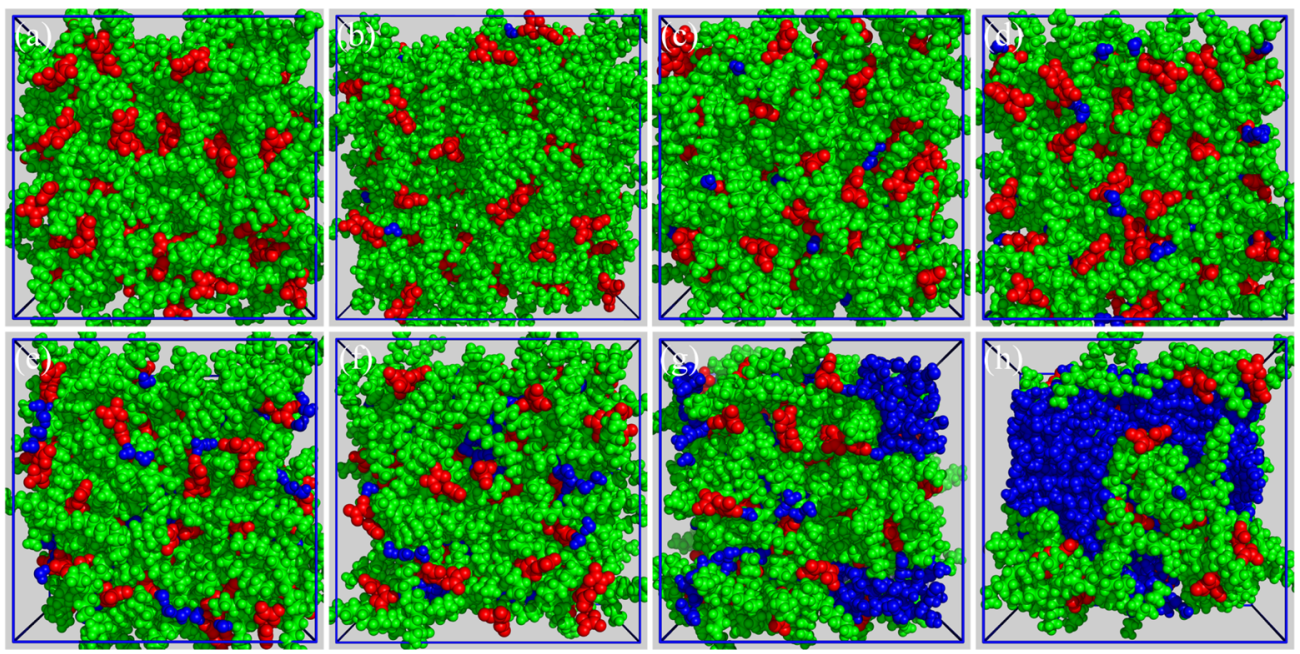

Figure 2. Representative snapshots of the $\left[\mathrm{P}_{6,6,6,14}\right][\mathrm{BOB}] \mathrm{IL} /$ water mixtures with water mole fractions of $x_{\text {water }}=(\mathrm{a}) 0.0,(\mathrm{~b}) 0.10,(\mathrm{c}) 0.33$, (d) 0.50 , (e) $0.67,(\mathrm{f}) 0.80,(\mathrm{~g}) 0.90$, and $(\mathrm{h}) 0.95$. The $\left[\mathrm{P}_{6,6,6,14}\right]$ cations, the $[\mathrm{BOB}]$ anions, and the water molecules in these $\mathrm{IL} /$ water mixtures are represented by green, red, and blue beads, respectively.

performed, and the simulation trajectories were recorded at intervals of $100 \mathrm{fs}$ for further analysis.

\section{STRUCTURAL ANALYSIS AND DISCUSSION}

Visualization of Microscopic Structure. Representative snapshots of $\left[\mathrm{P}_{6,6,6,14}\right][\mathrm{BOB}] \mathrm{IL} /$ water mixtures are presented in Figure 2, in which $\left[\mathrm{P}_{6,6,6,14}\right]$ cations, $[\mathrm{BOB}]$ anions, and water molecules are represented by green, red, and blue beads, respectively. In the neat IL simulation system (panel a), the microscopic liquid structure is characterized by a connected apolar network consisting of the alkyl chains of $\left[\mathrm{P}_{6,6,6,14}\right]$ cations and isolated polar domains composed of the central segments of $\left[\mathrm{P}_{6,6,6,14}\right]$ cations and $[\mathrm{BOB}]$ anions that are closely packed together by means of strong electrostatic interactions. Such a heterogeneous liquid structure is distinct from those formed in imidazolium-based ILs with short alkyl substituents, which is described by isolated apolar domains scattered in a connected polar network, or interpenetrating apolar and polar networks. ${ }^{44-48}$
With a gradual increase in the water concentration in IL/ water mixtures, the relative distribution of water molecules undergoes a particular transition from dispersed water monomers (panel b) to water dimers/trimers (panels $c$ and d), small clusters and chain-like structures (panels e and f) in isolated polar domains, large aggregates (panels $g$ and $h$ ), and eventually a water network in a water concentrated simulation system. Guided by these visual snapshots, a more systematic analysis of the microscopic liquid structure is performed to obtain a quantitative description of these IL/water mixtures as a function of the water concentration, and discussed in detail in the following subsections. Additionally, an extremely diluted $\left[\mathrm{P}_{6,6,6,14}\right][\mathrm{BOB}] \mathrm{IL} /$ water mixture with a water mole fraction of $x_{\text {water }}=0.99$ was further studied in the present work. ${ }^{49}$ A loose micelle-like aggregate characterized by a hydrophobic core composed of alkyl chains in $\left[\mathrm{P}_{6,6,6,14}\right]$ cations and a hydrophilic shell consisting of the central polar segments in $\left[\mathrm{P}_{6,6,6,14}\right]$ cations and $[\mathrm{BOB}]$ anions is formed and isolated in a highly branched water network in all frameworks of recorded 

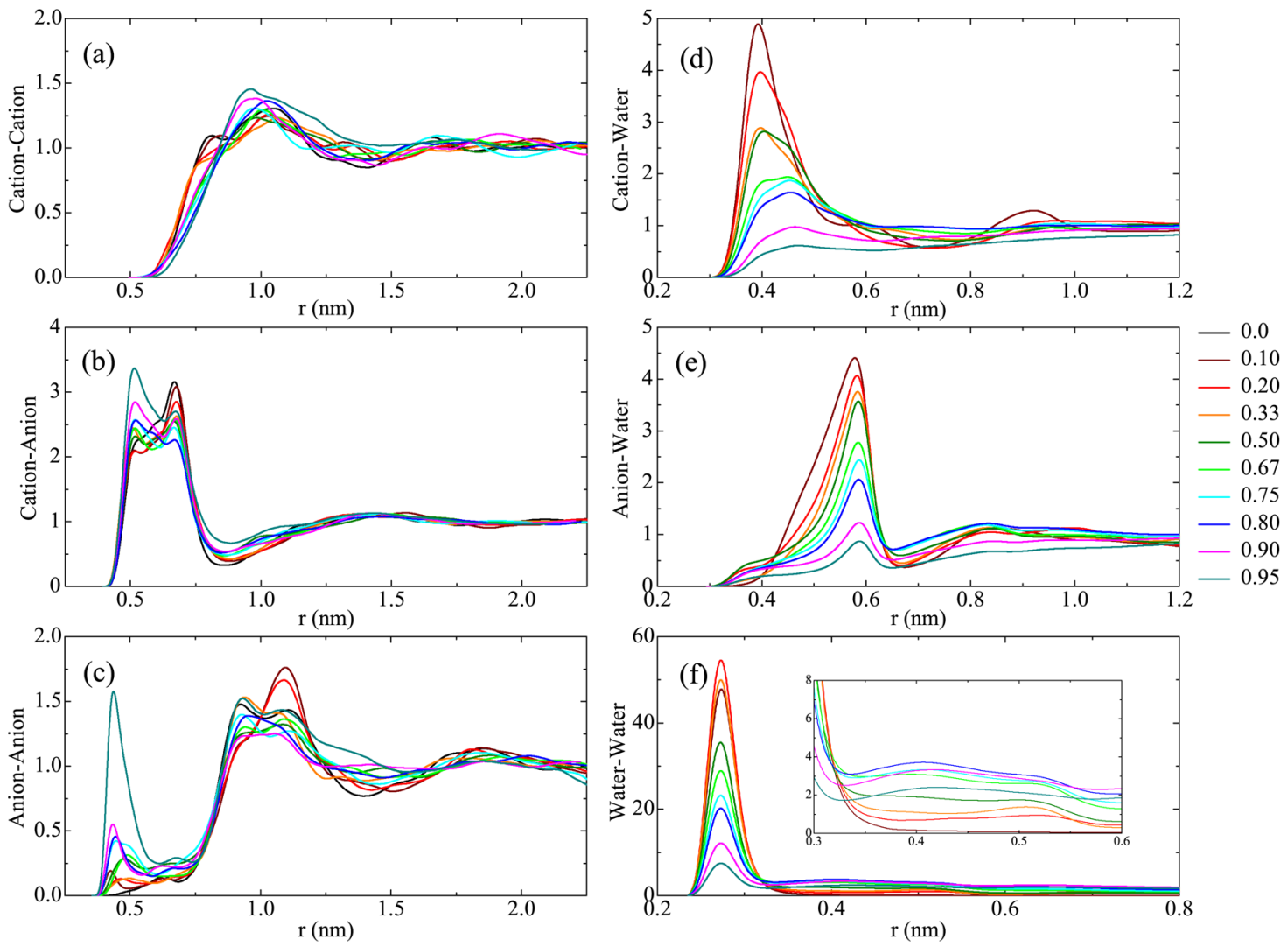

Figure 3. Radial distribution functions of (a) cation-cation, (b) cation-anion, (c) anion-anion, (d) cation-water, (e) anion-water, and (f) waterwater pairs calculated from the $\left[\mathrm{P}_{6,6,6,14}\right][\mathrm{BOB}] \mathrm{IL} /$ water mixtures as a function of water mole fractions.

simulation trajectories. This observation is a general feature of amphiphilic surfactant-like cations dissolved in water, such as the formation of micelles of imidazolium-based cations in diluted aqueous solutions, which has been extensively studied in previous work. ${ }^{50,51}$ The structural analysis of this diluted $\left[\mathrm{P}_{6,6,6,14}\right][\mathrm{BOB}] \mathrm{IL} /$ water mixture is not discussed in detail, but some simulation results are presented in the following subsections to show the general tendency of the evolution of microscopic ionic structure in the full water concentration range.

Microscopic Ionic Structure. The radial distribution function (RDF) quantifies the spatial correlation between a specific atom and other similar or dissimilar atoms in its surroundings, which is useful for the concretization of the microscopic ionic structure in $\mathrm{IL} /$ water mixtures. Figure 3 presents the site-site RDFs of cation-cation, cation-anion, anion-anion, cation-water, anion-water, and water-water pairs. The central $\mathrm{P}$ and $\mathrm{B}$ atoms in $\left[\mathrm{P}_{6,6,6,14}\right]$ cations and [BOB] anions, respectively, and the $\mathrm{OW}$ atoms in water molecules, as labeled in Figure 1, are taken as reference sites to calculate the corresponding site-site RDFs. In the meantime, the local liquid environment is qualitatively represented by examining the coordination number, which is the mean value of the number of a specific atom or molecule within a prescribed cutoff distance $r$ from a given reference atom or molecule. The coordination number is obtained by numerically integrating the corresponding RDF from zero to the first minimum, which represents the first solvation shell. The calculated coordination numbers from cation-anion, cation-water, anion-water, and water-water paired RDFs are shown in Figure 4.

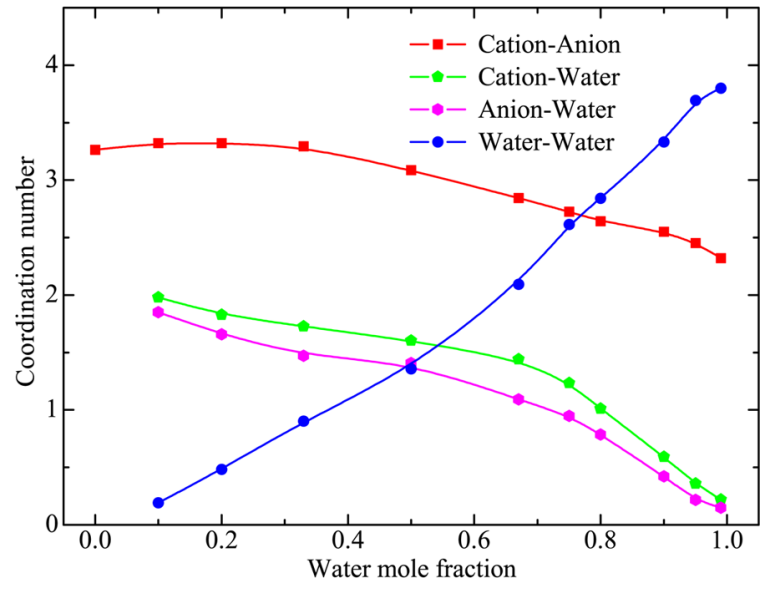

Figure 4. Mean values of the coordination numbers of cation-anion, cation-water, anion-water, and water-water pairs calculated from the $\left[\mathrm{P}_{6,6,6,14}\right][\mathrm{BOB}] \mathrm{IL} /$ water mixtures as a function of water mole fractions. The mean values of cation-water and anion-water pairs are obtained by averaging the corresponding total coordination numbers over the number of ion pairs in studied simulation systems.

In all studied IL/water mixtures with different water mole fractions, the cation-cation RDFs present very broad maxima centered at around $1.0 \mathrm{~nm}$ characterized by similar peak intensities, and the corresponding peak positions show little shift, indicating that the packing cationic structure, either in the neat IL simulation system or in the IL/water mixtures, is mainly determined by short-ranged interactions. However, significant changes are observed in the anion-anion paired RDFs, which are strongly dependent on the amount of water in the IL/water 

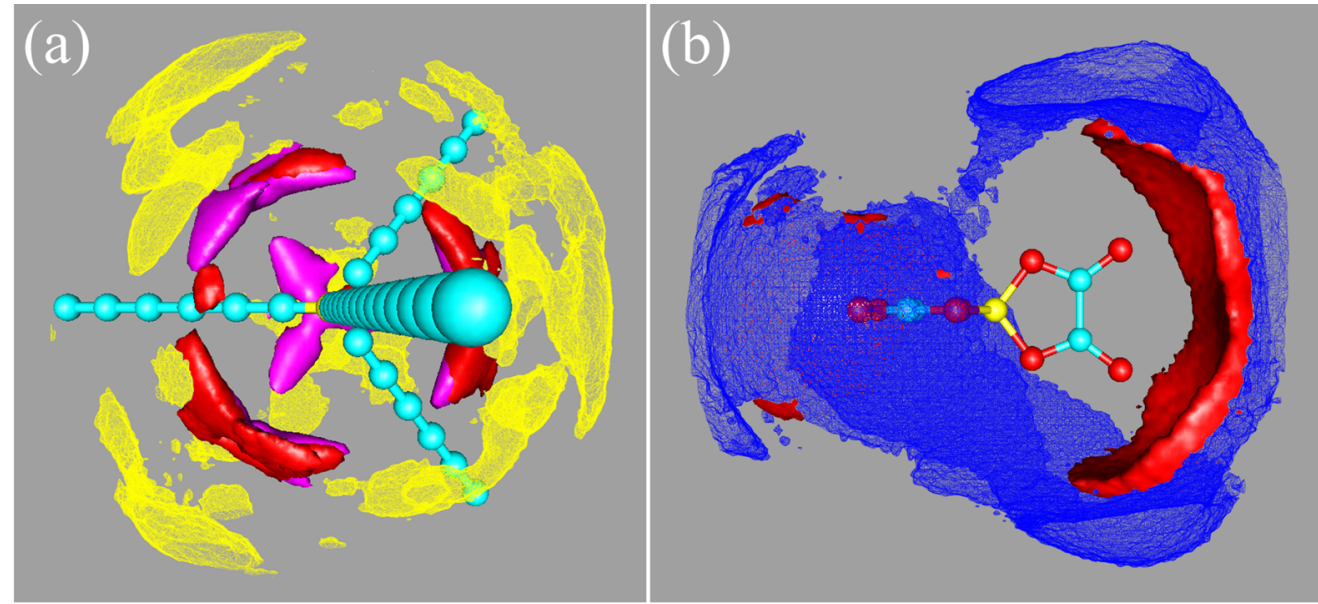

Figure 5. Three-dimensional probability distributions of specific atoms around a specific molecule in the $\left[\mathrm{P}_{6,6,6,14}\right][\mathrm{BOB}] \mathrm{IL} /$ water mixture with a water mole fraction of $x_{\text {water }}=0.50$. (a) The B (yellow meshed surface) and O (magenta solid surface) atoms in [BOB] anions, and the OW atoms (red solid surface) in water molecules around a $\left[\mathrm{P}_{6,6,6,14}\right]$ cation. The yellow, magenta, and red bounded contour surfaces are drawn at 5.0, 8.0, and 8.0 times of the average density of the corresponding atoms, respectively. The $\mathrm{P}$ and carbon atoms in the $\left[\mathrm{P}_{6,6,6,14}\right]$ cation are represented by yellow and cyan beads in the skeleton, respectively. (b) The $\mathrm{P}$ atoms (blue meshed surface) in $\left[\mathrm{P}_{6,6,6,14}\right]$ cations and the $\mathrm{OW}$ atoms (red solid surface) in water molecules around a $[\mathrm{BOB}]$ anion. The blue and red bounded contour surfaces are drawn at 5.5 and 5.5 times of the average density of the corresponding atoms, respectively.

mixtures. The spatial correlations between $[\mathrm{BOB}]$ anions in the neat IL simulation system reveal a sharp maximum at approximately $1.0 \mathrm{~nm}$, which is larger than the size of [BOB] anions, indicating the scattered distribution of $[\mathrm{BOB}]$ anions in the $\left[\mathrm{P}_{6,6,6,14}\right]$ cationic matrix. The addition of water molecules leads to new noticeable peaks located at around $0.44 \mathrm{~nm}$ in the anion-anion RDFs, and the corresponding peak intensities increase significantly (panel $\mathrm{c}$ of Figure 3), indicating the enhanced spatial correlations between $[\mathrm{BOB}]$ anions in water concentrated mixtures probably because of water molecules linking $[\mathrm{BOB}]$ anions via hydrogen bonds.

The enhanced correlations between $[\mathrm{BOB}]$ anions are also depicted in the cation-anion RDFs. It is clearly shown that the spatial correlations between $\left[\mathrm{P}_{6,6,6,14}\right]$ cations and $[\mathrm{BOB}]$ anions in all studied IL/water mixtures are characterized by conspicuous and bifurcated main peaks in the first solvation shell of the corresponding RDFs, which contribute to the most probable distribution of $\mathrm{B}$ atoms of $[\mathrm{BOB}]$ anions around $\mathrm{P}$ atoms of $\left[\mathrm{P}_{6,6,6,14}\right]$ cations and vice versa. When water molecules are added to the studied IL/water mixtures, a turnover is observed in the intensities of the bifurcated peaks, where the former one becomes stronger and the latter one becomes weaker, respectively. Such a striking observation indicates that the considerably enhanced spatial correlations between $\left[\mathrm{P}_{6,6,6,14}\right]$ cations and $[\mathrm{BOB}]$ anions gradually move to shorter distances as the water concentration increases, which is rationalized by a slightly rearranged distribution and orientation of ionic species in polar domains.

The integration of the first solvation shell of the cationanion RDFs reveals a slight decrease in the number of ionic groups in the nearest neighbor shell of a central counterion as the water concentration increases, as shown in Figure 4. The number of contact cation-anion pairs remains different from zero even in intermediate and water concentrated mixtures, something which is almost certainly due to the closely associated microscopic ionic structure predominantly driven by the strong electrostatic interactions between ionic species.

Microstructure between Ionic Species and Water. As the central polar segments of $\left[\mathrm{P}_{6,6,6,14}\right]$ cations always stay in a close proximity to neighboring $[\mathrm{BOB}]$ anions in the neat $\mathrm{IL}$ simulation system and the $\mathrm{IL} /$ water mixtures, it is reasonable to justify at least the existence of contact $\left[\mathrm{P}_{6,6,6,14}\right][\mathrm{BOB}]$ ion pairs. Then, a pertinent question to ask is why and how water molecules associate with these two ionic species in IL/water mixtures. ${ }^{52-54}$ Panels $d$ and e of Figure 3 present the RDFs of water molecules relative to $\left[\mathrm{P}_{6,6,6,14}\right]$ cations and $[\mathrm{BOB}]$ anions in all studied $\mathrm{IL} /$ water mixtures, respectively. The main feature of the cation-water and the anion-water RDFs is that the first peaks are sharper and higher in IL/water mixtures with less water. However, the spatial correlations between the respective ionic species and water molecules exhibit significant variations over the full water concentration range.

In the cation-water RDFs, the first peak intensities undergo progressive reduction, and the corresponding peak positions gradually shift to larger distances as the water concentration increases. However, the influence of the water concentration on the spatial correlations between $[\mathrm{BOB}]$ anions and water molecules is only recorded in the peak intensities of the anionwater RDFs, as presented in panel e of Figure 3. The main peak positions of the anion-water RDFs are essentially unchanged in all studied IL/water mixtures, indicating that the radius of the first solvation shell is substantially independent of the number of water molecules in IL/water mixtures, and the interactions between $[\mathrm{BOB}]$ anions and water molecules are limited to specific atoms.

The reduction of the first peak intensities in the cationwater and anion-water RDFs indicates a decreased tendency of water molecules to coordinate with $\left[\mathrm{P}_{6,6,6,14}\right]$ cations and $[\mathrm{BOB}]$ anions with the increase in water mole fractions in IL/ water mixtures, as manifested in the corresponding coordination numbers shown in Figure 4. Such a tendency may be attributed to the saturated distribution of water molecules between contact neighboring $\left[\mathrm{P}_{6,6,6,14}\right][\mathrm{BOB}]$ ion pairs. As more water molecules are introduced in $\mathrm{IL} /$ water mixtures, the additional water molecules tend to aggregate and form distinct water morphologies, such as small clusters, chain-like structures, and large aggregates, as clearly visible in Figure 2. 
Comparing with the cation-anion RDFs, it is observed that the most probable distribution of water molecules is located in the middle of the bifurcated main peaks, indicating that water molecules are optimally incorporated in cavities between neighboring ionic species and are much closer to the central $\mathrm{P}$ atoms of $\left[\mathrm{P}_{6,6,6,14}\right]$ cations than to the $\mathrm{B}$ atoms of $[\mathrm{BOB}]$ anions. Concerning the cation-anion, cation-water, and anion-water RDFs, we can specify that the microscopic liquid structure and the relative distribution of water molecules in the local ionic organization are determined by cation-water-anion triple or multiple complexes in IL/water mixtures.

In addition to the spherically averaged RDF and the coordination number, the microscopic liquid structure in IL/ water mixtures is further illustrated by the spatial distribution function (SDF), giving the probability of finding an atom around a specific molecule in three-dimensional space. ${ }^{40,50,55,56}$ In the present work, all SDFs are well visualized by the gOpenMol package. ${ }^{57,58}$

Figure 5 presents typical SDFs calculated from the IL/water mixture with a water mole fraction of $x_{\text {water }}=0.50$, in which the number of $\left[\mathrm{P}_{6,6,6,14}\right][\mathrm{BOB}]$ ion pairs is equal to that of water molecules. Panel a shows the SDFs of $\mathrm{B}$ and $\mathrm{O}$ atoms in [BOB] anions, as well as the $\mathrm{OW}$ atoms in water molecules around a $\left[\mathrm{P}_{6,6,6,14}\right]$ cation. The $\mathrm{B}$ and $\mathrm{O}$ atoms coordinate with the central polar segment of the $\left[\mathrm{P}_{6,6,6,14}\right]$ cation in the tetrahedral region due to the strong electrostatic interactions between central polar segments of the $\left[\mathrm{P}_{6,6,6,14}\right]$ cation and the $[\mathrm{BOB}]$ anion and the strong steric effect of four alkyl substituents in the $\left[\mathrm{P}_{6,6,6,14}\right]$ cation. The most probable distribution of OW atoms is located between that of $\mathrm{B}$ atoms and the central segment in the $\left[\mathrm{P}_{6,6,6,14}\right]$ cation, and is comparable with the most probable distribution of $\mathrm{O}$ atoms in [BOB] anions. Such striking spatial distributions of $\mathrm{B}$ and $\mathrm{OW}$ atoms, which contribute to the first solvation shell in the cation-anion and cation-water RDFs, further verify that water molecules are embedded in cavities between neighboring ionic species and are strongly associated with $\left[\mathrm{P}_{6,6,6,14}\right]$ cations and $[\mathrm{BOB}]$ anions through cation-water-anion triple complexes. The local microscopic liquid structure is mediated by the oxygen atoms, either of $[\mathrm{BOB}]$ anions in the neat IL simulation system or of $[\mathrm{BOB}]$ anions and of water molecules in the $\mathrm{IL} /$ water mixtures.

Panel $b$ of Figure 5 presents the SDFs of $\mathrm{P}$ atoms in $\left[\mathrm{P}_{6,6,6,14}\right]$ cations and $\mathrm{OW}$ atoms around $\mathrm{a}[\mathrm{BOB}]$ anion in the $\mathrm{IL} /$ water mixture with a water mole fraction of $x_{\text {water }}=0.50$. The $\mathrm{P}$ atoms reside around the equatorial positions of the two fivemembered rings in the $[\mathrm{BOB}]$ anion, and the corresponding distribution is characterized by broadened and symmetric but less site-specific features. The effect of the water concentration on the SDF of $\mathrm{P}$ atoms around the $[\mathrm{BOB}]$ anion is negligible (data not shown) due to the strong electrostatic interactions between the central segments in ionic species. The most probable distribution of $\mathrm{OW}$ atoms is exclusively constrained along the $\mathrm{C}=\mathrm{O}$ vectors in the $[\mathrm{BOB}]$ anion, which facilitates the formation of hydrogen bonds between [BOB] anions and water molecules and will be discussed in the following subsection. A decrease in the water concentration in $\mathrm{IL} /$ water mixtures leads to an enhanced three-dimensional probability distribution of $\mathrm{OW}$ atoms around the [BOB] anion and vice versa (data not shown), as verified using the corresponding anion-water RDFs.

Clustering of Water Molecules. The distinct interactions between ionic species and water molecules not only disturb the microscopic liquid structure between $\left[\mathrm{P}_{6,6,6,14}\right]$ cations and
$[\mathrm{BOB}]$ anions but also affect the relative distribution of water molecules in the IL matrix. Panel $\mathrm{f}$ of Figure 3 illustrates that the water-water RDFs exhibit similar weakened spatial correlations in IL/water mixtures with increased water concentration. A quantitative change observed in the waterwater RDFs is that the region beyond the first peak gradually fills up as the water concentration increases, suggesting the aggregation of additional water molecules and the formation of small clusters or chain-like aggregates. In IL/water mixtures with water mole fractions of $0.20<x_{\text {water }}<0.50$, the growth of a secondary peak at approximately $0.51 \mathrm{~nm}$ in the water-water RDFs contributes to the establishment of a secondary solvation shell of water molecules around a central one, indicating a forming of water clusters in this region as the water concentration increases. ${ }^{59}$

To further quantify the extent of microscopic water structure changes as the water concentration increases, the probability distribution of the water cluster sizes is analyzed by monitoring the connectivity of water molecules. Two water molecules are considered to be mutually "connected" according to simplified geometric criterion; ${ }^{60}$ i.e., the $\mathrm{OW}-\mathrm{OW}$ distance is less than $0.35 \mathrm{~nm}$, which corresponds to the first minimum in the water-water RDFs. If there is no connectivity of a water molecule with any other water molecules, it is a water monomer, or "isolated" water molecule. If two water molecules are connected without any connectivity with other water molecules, a water dimer is formed. Small water clusters, such as trimers, tetramers, etc., can also be discriminated using a similar analysis. Meanwhile, a water network is formed when a water cluster is large enough to percolate from one simulation cell to its image. In the present work, the probability distribution of water cluster sizes in IL/water mixtures with water mole fractions of $x_{\text {water }} \leq 0.80$ is obtained by forming the ensemble average, and presented in Figure 6.

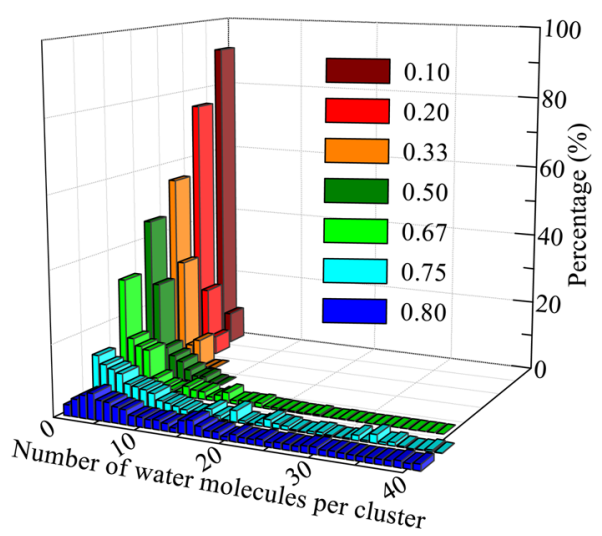

Figure 6. Probability distribution of water cluster sizes in the $\left[\mathrm{P}_{6,6,6,14}\right][\mathrm{BOB}] \mathrm{IL} /$ water mixtures with water mole fractions of $x_{\text {water }}$ $\leq 0.80$ obtained from the present atomistic simulations.

In IL/water mixtures with a low water concentration, such as $x_{\text {water }}=0.10$ corresponding to 11 water molecules per 96 $\left[\mathrm{P}_{6,6,6,14}\right][\mathrm{BOB}]$ ion pairs, all water molecules tend to be strongly isolated as dispersed water monomers. These dispersed water monomers coordinate simultaneously with neighboring $\left[\mathrm{P}_{6,6,6,14}\right]$ cations and $[\mathrm{BOB}]$ anions through cation-water-anion triple complexes.

As the water concentration increases in the IL/water mixtures, such as $0.20<x_{\text {water }}<0.50$, the number of close water contacts grows at a slower rate than the number of water 

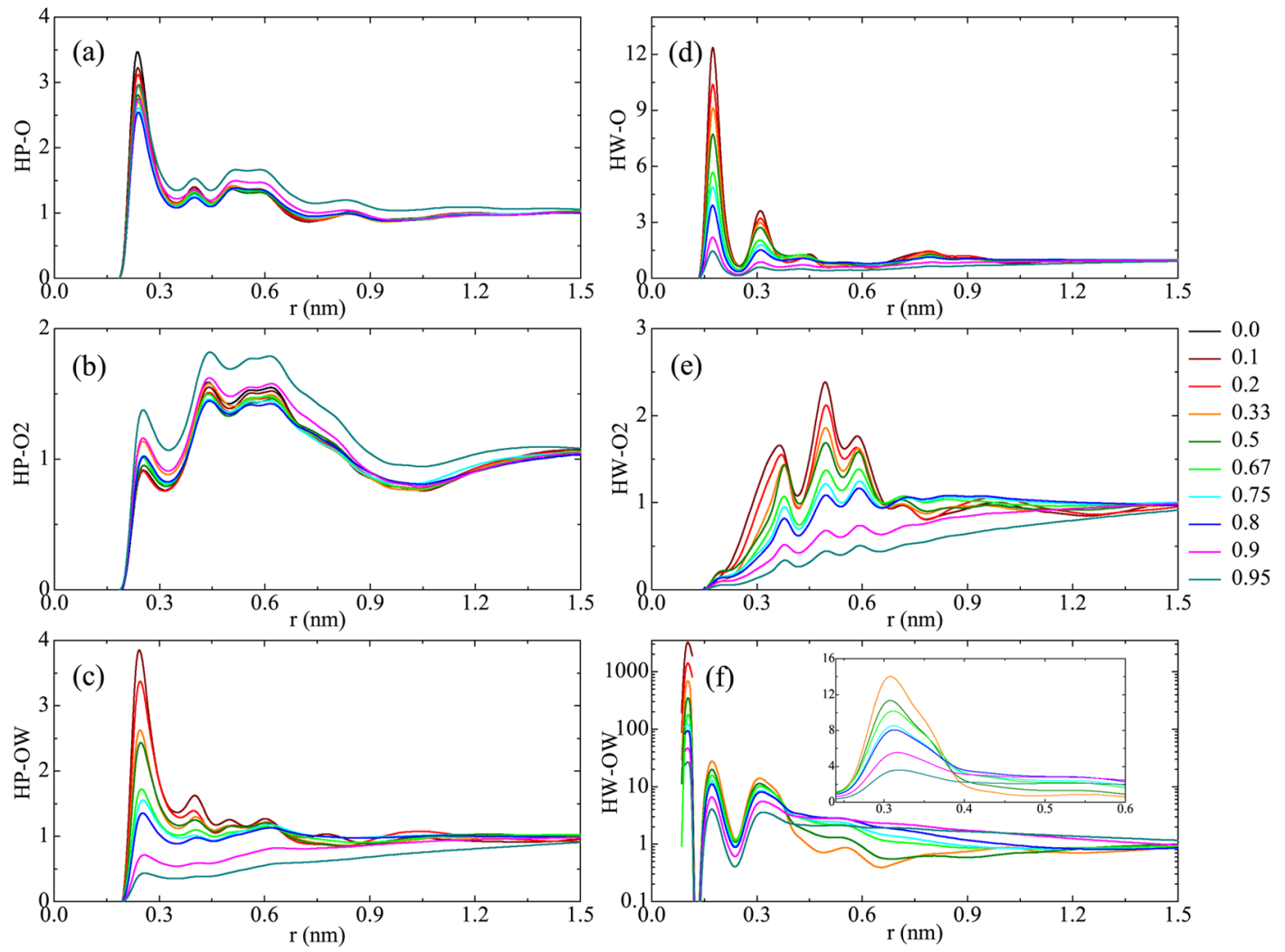

Figure 7. Radial distribution functions of hydrogen atoms (the $\mathrm{HP}$ atoms in $\left[\mathrm{P}_{6,6,6,14}\right]$ cations and the $\mathrm{HW}$ atoms in water molecules) and oxygen atoms (the $\mathrm{O}$ and $\mathrm{O} 2$ atoms in $[\mathrm{BOB}]$ anions and the $\mathrm{OW}$ atoms in water molecules) for the $\left[\mathrm{P}_{6,6,6,14}\right][\mathrm{BOB}] \mathrm{IL} /$ water mixtures with varied water mole fractions: (a) HP-O, (b) HP-O2, (c) HP-OW, (d) HW-O, (e) HW-O2, and (f) HW-OW.

molecules, which is consistent with the diminished first peak intensities in the water-water RDFs. Small water clusters, such as water dimers and trimers, are present, but no large water aggregates are formed in these IL/water mixtures. It can be shown that even more than $50 \%$ of the added water molecules are completely dispersed in the IL/water mixture with a water mole fraction of $x_{\text {water }}=0.50$.

For the $\mathrm{IL} /$ water mixtures with intermediate water mole fractions of $0.67<x_{\text {water }}<0.80$, there is a significant jump in the probability distribution of water cluster sizes. Large water aggregates appear to dominate the size distribution due to the fact that there is not a sufficient void space between neighboring ionic species to accommodate more water molecules. Besides the formation of compact water clusters, a striking observation is the emergence of the chain-like water structures in IL/water mixtures, which connect more ionic species between the isolated polar domains, but the apolar network is not explicitly altered.

Further progressive dilution of IL/water mixtures leads to the buildup of a water network, in which all water molecules dynamically percolate throughout the entire simulation cell. In the meantime, the corresponding simulation systems undergo a transformation from IL-rich mixtures to those characterized by interpenetrating polar and apolar networks and eventually to aqueous solutions of IL in which micelle-like aggregates are formed and scattered in a highly branched water network. Therefore, the analysis of the probability distribution of water cluster sizes is not shown for the IL/water mixtures with water mole fractions of $0.80<x_{\text {water }}<1.0$.

The evolution of the water cluster size distribution in IL/ water mixtures is further reinforced by the analysis of the mean value of the coordination number of water molecules, as shown in Figure 4. There are approximately 3.8 water molecules around a central water molecule in the $\mathrm{IL} /$ water mixture with a water mole fraction of $x_{\text {water }}=0.99$, consistent with that reported for/on bulk liquid water. ${ }^{61}$

Hydrogen Bond Network. It is well-known that in the neat ILs the electrostatic interactions between ionic species are the dominating interactions, which directly contribute to the close packing of ions in ionic structures. Furthermore, the thermodynamic, transport, and other properties of ILs are also strongly dependent on the electrostatic interactions between ionic species. In IL/water mixtures, not only the atom-centered electrostatic interactions but also the hydrogen bonded interactions play an important role in determining IL-related properties. ${ }^{50,51,53,54}$ Herein, we explore the formation of hydrogen bonds between ionic species and water molecules in the IL/water mixtures with varied water mole fractions.

The formation of hydrogen bonds is defined by a simple geometric criterion; i.e., the radial distance between the labeled hydrogen atom and the acceptor atom is equal to or less than $0.25 \mathrm{~nm}$, and the angle of donor-hydrogen $\cdots$ acceptor is larger than $150^{\circ} .{ }^{60}$ In $\mathrm{IL} /$ water mixtures, the possible hydrogen atoms are the $\mathrm{HP}$ atoms in $\mathrm{CH}_{2}$ groups connected to the central $\mathrm{P}$ atoms in $\left[\mathrm{P}_{6,6,6,14}\right]$ cations and the $\mathrm{HW}$ atoms in water molecules, and the available acceptor atoms include all oxygen atoms in $[\mathrm{BOB}]$ anions (the $\mathrm{O}$ and $\mathrm{O} 2$ atoms), and the $\mathrm{OW}$ atoms in water molecules, respectively, as labeled in Figure 1. The radial distances between respective hydrogen atoms and acceptor oxygen atoms are analyzed by computing the corresponding RDFs which are shown in Figure 7. 
Panels a-c of Figure 7 present the RDFs between HP atoms and three types of oxygen atoms in IL/water mixtures with varied water concentrations, respectively. The first maxima are located at $0.24,0.25$, and $0.24 \mathrm{~nm}$ in the HP-O, HP-O2, and $\mathrm{HP}-\mathrm{OW}$ paired RDFs, respectively, indicating that $\mathrm{HP}$ atoms are primarily hydrogen bonded to all three types of oxygen atoms in the studied IL/water mixtures. However, a distinct change is observed in the first peak intensities of these three RDFs. Both the HP-O and the HP-OW RDFs exhibit decreased first peak intensities as a function of the water concentration, whereas an opposite tendency is observed in the HP-O2 RDFs, in which the first peak heights become considerably more intense as the water concentration increases. In IL/water mixtures with less water, such as $x_{\text {water }}<0.50$, the comparable first peak intensities in the HP-O and HP-OW RDFs suggest a similar coordination of $\mathrm{O}$ and $\mathrm{OW}$ atoms to $\mathrm{HP}$ atoms, and the coordination ability of the three types of oxygen atoms follows the order of $\mathrm{O} \approx \mathrm{OW}>\mathrm{O} 2$. For $\mathrm{IL} /$ water mixtures with intermediate water mole fractions of $0.67<x_{\text {water }}<0.80$, a strong decrease in the first peak intensities of the HP-OW $\mathrm{RDF}$ reflects a reduction in the hydrogen bonded interactions between OW and HP atoms, leading to the coordination behavior of three types of oxygen atoms described by $\mathrm{O}>\mathrm{OW}$ $>\mathrm{O} 2$. An increase in the water concentration in $\mathrm{IL} /$ water mixtures leads to a further decrease in the first peak heights in the HP-OW RDFs. The coordination tendency of the three types of oxygen atoms is characterized by $\mathrm{O}>\mathrm{O} 2>\mathrm{OW}$ in water concentrated mixtures.

The dependence of the radial distances between HW atoms and the three types of oxygen atoms in $\mathrm{IL} /$ water mixtures on the water mole fractions are presented in panels $\mathrm{d}-\mathrm{f}$ of Figure 7, respectively. Comparing with the $\mathrm{HW}-\mathrm{O}$ RDFs, the pronounced peak intensities in the HW-OW RDFs indicate that $\mathrm{OW}$ atoms are stronger hydrogen bond acceptors than $\mathrm{O}$ atoms in the $[\mathrm{BOB}]$ anions to neighboring water molecules over the whole water concentration range. However, in the $\mathrm{HW}-\mathrm{O} 2$ RDFs, all triple peak positions, which correspond to the most probable distribution of $\mathrm{HW}$ atoms around $\mathrm{O} 2$ atoms in the $[\mathrm{BOB}]$ anion, are beyond $0.25 \mathrm{~nm}$, indicating that $\mathrm{HW}$ and $\mathrm{O} 2$ atoms are not hydrogen bonded to each other in any of these studied IL/water mixtures.

Due to the relative central position of HP atoms and the large volume of $\left[\mathrm{P}_{6,6,6,14}\right]$ cations, it is difficult to visualize the three-dimensional probability distributions of three types of oxygen atoms around HP atoms. Conversely, we plot the SDF of $\mathrm{HP}$ atoms around a $[\mathrm{BOB}]$ anion in the $\mathrm{IL} /$ water mixture with a water mole fraction of $x_{\text {water }}=0.50$ and present it in panel a of Figure 8, in which the spatial distribution of $\mathrm{P}$ atoms is also presented as a reference. It is clearly shown that the most probable distribution of HP atoms occupies the equatorial positions of the two five-membered rings in the depicted [BOB] anion, indicating that HP atoms are strongly hydrogen bonded to both $\mathrm{O}$ and $\mathrm{O} 2$ atoms in [BOB] anions. The influence of the water concentration on the SDFs of $\mathrm{P}$ and HP atoms around a $[\mathrm{BOB}]$ anion is negligible (data not shown), something that can be attributed to the strong electrostatic interactions between central polar segments in ionic species and the favorable hydrogen bonded interactions, respectively.

Similarly, the SDF of HW atoms around a [BOB] anion in the IL/water mixture with a water mole fraction of $x_{\text {water }}=0.50$ is presented in panel b of Figure 8, in which the SDF of OW atoms is also shown for a better comparison. The dominant isodensity surface of $\mathrm{HW}$ atoms is particularly concentrated
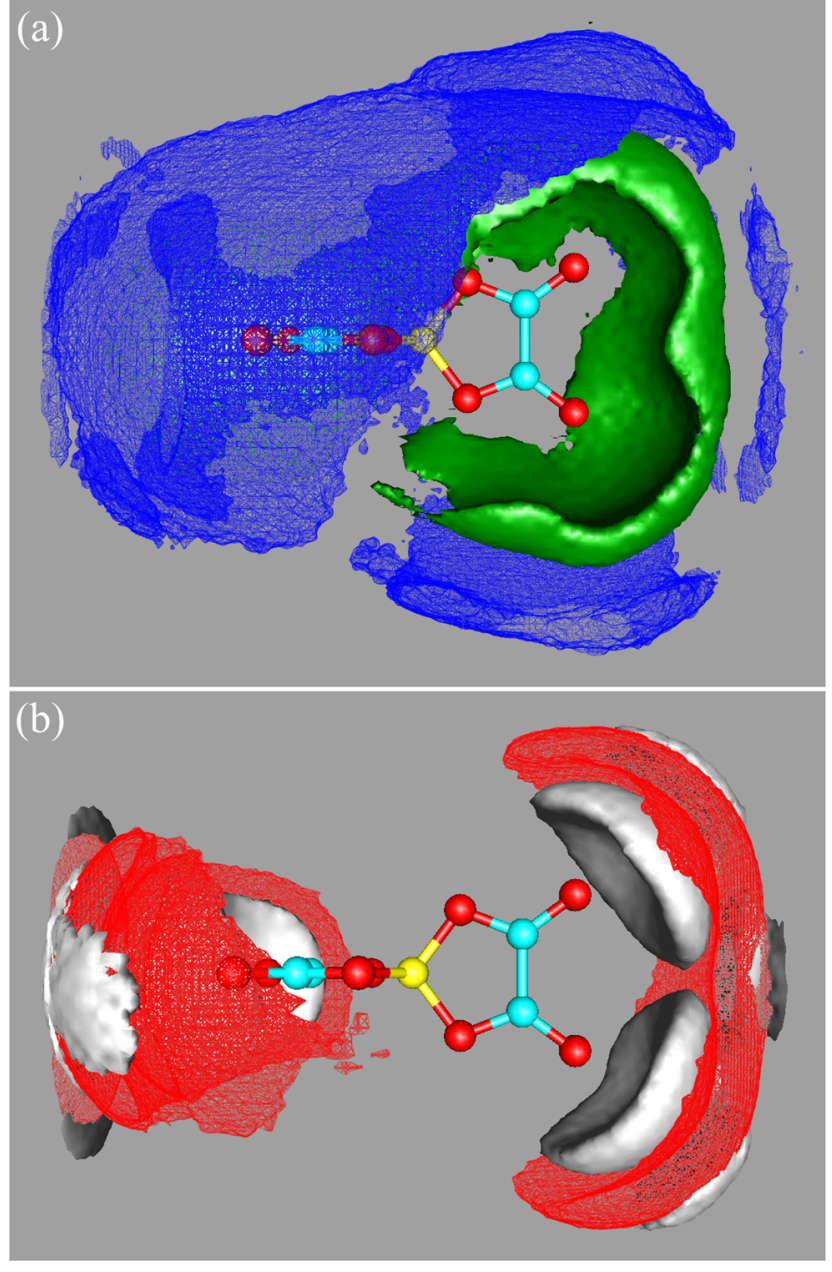

Figure 8. Three-dimensional probability distributions of specific atoms around a $[\mathrm{BOB}]$ anion in the $\left[\mathrm{P}_{6,6,6,14}\right][\mathrm{BOB}] \mathrm{IL} /$ water mixture with a water mole fraction of $x_{\text {water }}=0.50$. (a) The P (blue meshed surface) and HP (olive solid surface) atoms in $\left[\mathrm{P}_{6,6,6,14}\right]$ cations around the $[\mathrm{BOB}]$ anion. The blue and olive bounded contour surfaces are drawn at 5.5 and 2.8 times of the average density of the corresponding atoms, respectively. (b) The OW (red meshed surface) and HW (white solid surface) atoms in water molecules around the [BOB] anion. The red and white bounded contour surfaces are drawn at 5.0 and 5.0 times of the average density of the corresponding atoms, respectively.

along the $\mathrm{C}=\mathrm{O}$ vectors, which is consistent with the strong spatial correlations present in the HW-O RDFs, indicating that the hydrogen bonded interactions between [BOB] anions and water molecules are specifically localized around $\mathrm{O}$ atoms in the displayed $[\mathrm{BOB}]$ anion. The region adjacent to $\mathrm{O} 2$ atoms in the $[\mathrm{BOB}]$ anion is devoid of HW atoms, as expected, which mirrors the distinct $\mathrm{HW}-\mathrm{O} 2 \mathrm{RDF}$ shown in panel e of Figure 7. A decrease in the water concentration in IL/water mixtures leads to enhanced spatial probability distributions of OW and $\mathrm{HW}$ atoms around the $[\mathrm{BOB}]$ anion and vice versa (data not shown), as verified by similar tendencies present in the corresponding RDFs.

Three-dimensional organizations of the $\mathrm{P}$ and $\mathrm{HP}$ atoms, as well as the OW and HW atoms, around a selected water molecule are presented in Figure 9. Not surprisingly, HP atoms are preferentially oriented toward the lone electron pairs in the water molecule. The spatial probability distribution of $\mathrm{HP}$ atoms around a water molecule is broad and symmetric, and consistent with that of HW atoms, but is strongly dependent on 


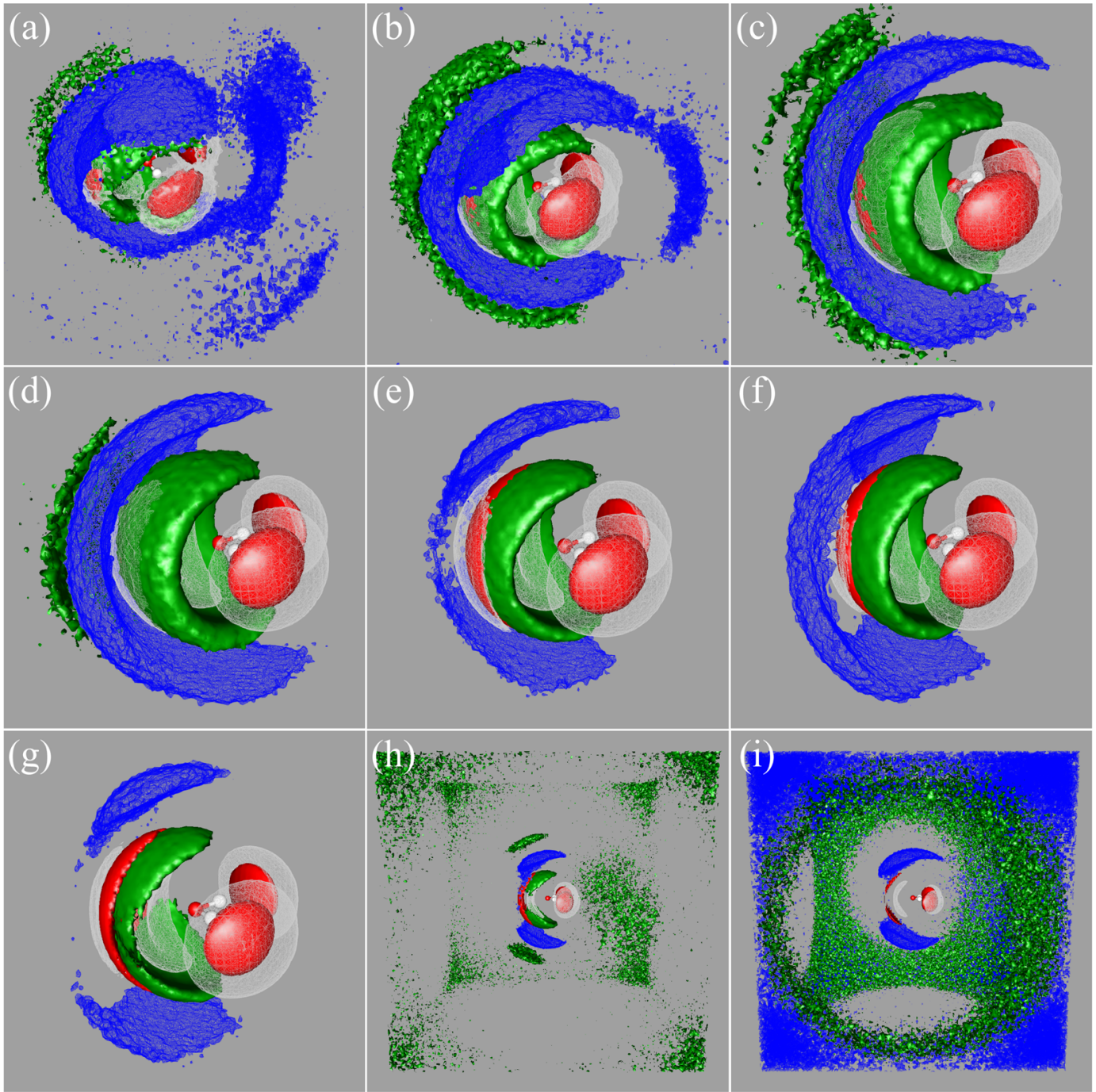

Figure 9. Three-dimensional probability distributions of the $\mathrm{P}$ (blue meshed surface) and HP (olive solid surface) atoms in $\left[\mathrm{P}_{6,6,6,14}\right]$ cations and the OW (red solid surface) and HW (white meshed surface) atoms in water molecules around a selected water molecule in IL/water mixtures with varied water mole fractions. All bounded contour surfaces are drawn at $\left(a_{\mathrm{P}}, a_{\mathrm{HP}}, a_{\mathrm{OW}}, a_{\mathrm{HW}}\right)$ times of the average density of the corresponding atoms, respectively. (a) $x_{\text {water }}=0.10$ with $(5.0,2.7,20.0,10.0)$, (b) $x_{\text {water }}=0.20$ with $(5.0,2.0,20.0,10.0),(\mathrm{c}) x_{\text {water }}=0.33$ with $(4.0,2.0,20.0,10.0),(\mathrm{d})$ $x_{\text {water }}=0.50$ with $(4.0,2.0,20.0,10.0)$, (e) $x_{\text {water }}=0.67$ with $(4.0,2.0,20.0,10.0),(\mathrm{f}) x_{\text {water }}=0.75$ with $(3.5,2.0,20.0,10.0),(\mathrm{g}) x_{\text {water }}=0.80$ with $(3.5,2.0,20.0,10.0)$, (h) $x_{\text {water }}=0.90$ with $(1.8,1.1,15.0,10.0)$, and (i) $x_{\text {water }}=0.95$ with $(1.0,0.9,13.0,10.0)$.

the water concentration in $\mathrm{IL} /$ water mixtures. It is clearly shown that the local water environment progressively loses neighboring $\left[\mathrm{P}_{6,6,6,14}\right]$ cations as the water concentration gradually increases. In the IL/water mixture with a water mole fraction of $x_{\text {water }}=0.95$, the spatial probability distribution of HP atoms is quite isotropic around the water molecule, and it is consequently different from the specific SDFs of OW and HW atoms around the water molecule. This is expected and reasonable as a branched water network is formed in this mixture, and therefore, the local water structure resembles that of bulk liquid water. The formation of hydrogen bonds between neighboring water molecules is exclusively localized along the OW-HW vectors and the lone electron pairs in the central water molecule, which is consistent with that described in bulk liquid water reported in the previous work. ${ }^{61}$

The mean values of the numbers of hydrogen bonds formed between ionic species and water molecules in the full water concentration range are presented in Figure 10. In all studied $\mathrm{IL} /$ water mixtures, the mean value of the number of hydrogen bonds formed between $\left[\mathrm{P}_{6,6,6,14}\right]$ cations and $[\mathrm{BOB}]$ anions monotonically and slowly decreases as a function of the water concentration. In IL-rich mixtures, i.e. with a water mole fraction of $x_{\text {water }} \leq 0.80$, the linear decrease in the mean value of the number of hydrogen bonds indicates that the orientation and distribution of two ionic species are slightly rearranged so as to accommodate more water molecules in their vicinities. The formation of cation-anion hydrogen bonds is still frequent in extremely diluted IL/water mixtures due to the formation of micelle-like aggregates in a highly branched water network, in which the central polar segments in $\left[\mathrm{P}_{6,6,6,14}\right]$ cations and [BOB] anions are strongly coupled together and form a polar shell, which facilitates their hydrogen bonded interactions.

The numbers of hydrogen bonds formed in the cationwater, anion-water, and water-water pairs increase monotonically and almost linearly as a function of the water concentration. In IL/water mixtures with less water, almost all water molecules are dispersed, acting like ideal gas molecules in IL matrix. Thus, the possibility of two water molecules being 


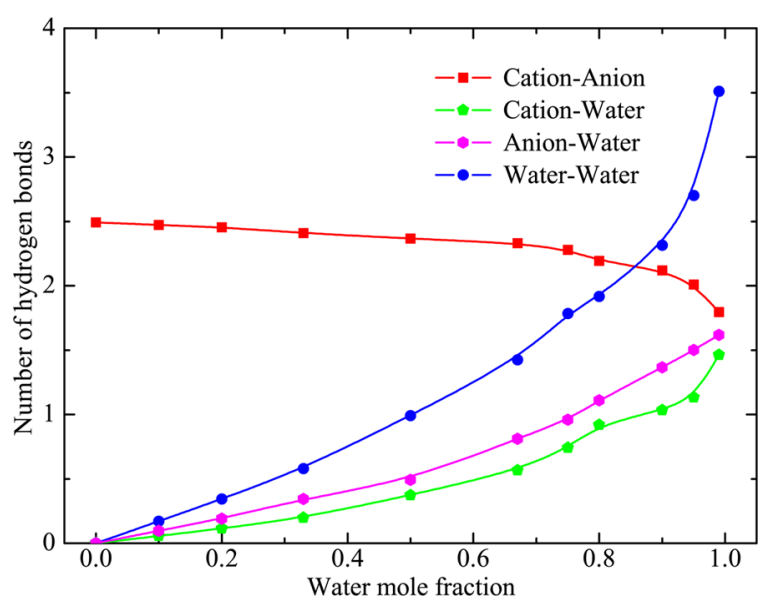

Figure 10. Mean values of the numbers of hydrogen bonds formed in the cation-anion, cation-water, anion-water, and water-water pairs calculated from the $\left[\mathrm{P}_{6,6,6,14}\right][\mathrm{BOB}] \mathrm{IL} /$ water mixtures as a function of water mole fractions. The mean values of cation-water and anionwater pairs are obtained by averaging the corresponding total hydrogen bonds over the number of ion pairs in studied simulation systems.

close enough to form a hydrogen bond is approximately proportional to water mole fractions in $\mathrm{IL} /$ water mixtures, as well as the formation of hydrogen bonds in the cation-water and anion-water pairs. This linear behavior is clearly discerned in Figure 10, and in fact, it extends reasonably well up to IL/ water mixtures with intermediate water mole fractions, i.e. $x_{\text {water }}$ $=0.80$. Further progressive dilution of IL/water mixtures leads to a distinct increase in the number of hydrogen bonds between water molecules, which may contribute to a microscopic liquid structural transition from isolated polar domains scattered in an apolar network to bicontinuous apolar and polar networks, and eventually to a highly branched water network. In extremely diluted IL/water mixtures, i.e. with a water mole fraction of $x_{\text {water }}=0.99$, there are approximately 3.5 hydrogen bonds per water molecule formed with neighboring water molecules, which is consistent with that previously reported for a bulk liquid water. ${ }^{61}$

Combining the mean values of the numbers of hydrogen bonds in the cation-anion, cation-water, anion-water, and water-water pairs shown in Figure 10 and the RDFs between hydrogen atoms and three types of oxygen atoms presented in Figure 7 , it is clear that water molecules are less selective in choosing their interaction partners, either in IL-rich or in water concentrated mixtures, probably because of their small molecular size and their dual nature in hydrogen bonded interactions. ${ }^{53}$ Water molecules act as hydrogen bond donors toward neighboring [BOB] anions and other water molecules and as hydrogen bond acceptors to the $\mathrm{HP}$ atoms in $\left[\mathrm{P}_{6,6,6,14}\right]$ cations and to the HW atoms in other water molecules, whereas $\left[\mathrm{P}_{6,6,6,14}\right]$ cations and $[\mathrm{BOB}]$ anions are restricted to their single role of hydrogen bond donors and acceptors, respectively.

Mechanism of Organization. To better quantify the evolution of the microscopic liquid structure in all studied IL/ water mixtures, we have performed a further analysis of $\left[\mathrm{P}_{6,6,6,14}\right]$ cationic aggregates and the intrinsic interactions between $\left[\mathrm{P}_{6,6,6,14}\right]$ cations and water molecules in the whole water concentration range. The $\left[\mathrm{P}_{6,6,6,14}\right]$ cation consists of two different parts: the hydrophilic core, including the $\mathrm{P}$ atom with directly connected four $\mathrm{CH}_{2}$ methylene groups, and the hydrophobic shell consisting of four volume-occupying alkyl chains, and hence it presents amphiphilic surfactant-like behavior in aqueous solutions. The introduction of water molecules in $\left[\mathrm{P}_{6,6,6,14}\right][\mathrm{BOB}] \mathrm{IL}$, on one hand, leads to the enhanced aggregation of these alkyl chains due to their hydrophobic nature, and on the other hand, water molecules tend to coordinate with HP atoms due to the favorable hydrogen bonded interactions, and preferentially associate with $\mathrm{P}$ atoms because of the strong electrostatic interactions.

Figure 11 presents the RDFs of terminal carbon atoms of the hexyl and tetradecyl chains in $\left[\mathrm{P}_{6,6,6,14}\right]$ cations. In $\mathrm{IL} /$ water

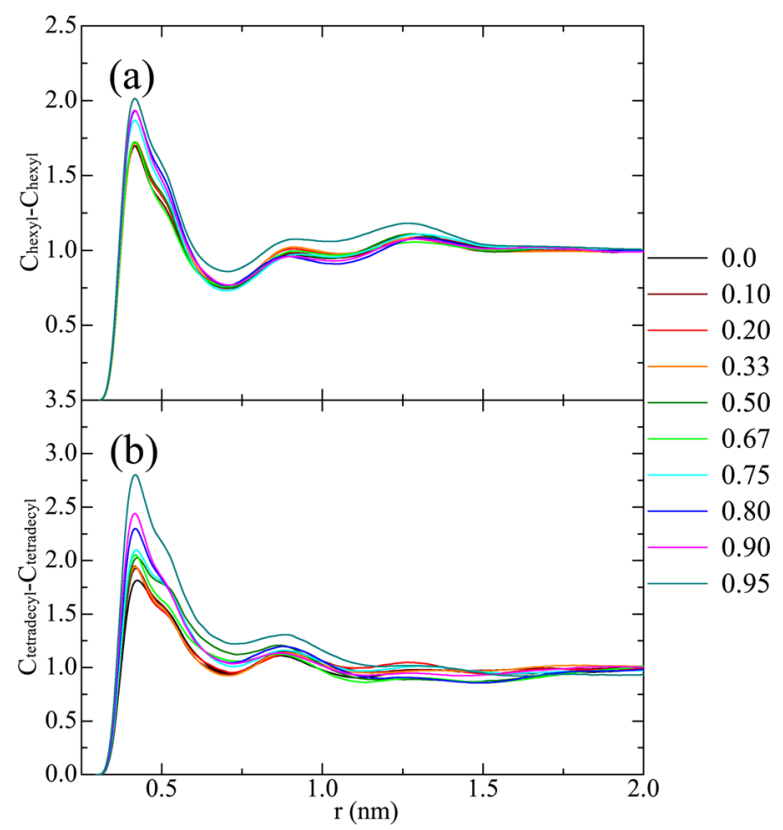

Figure 11. Radial distribution functions of the terminal carbon atoms in alkyl chains of $\left[\mathrm{P}_{6,6,6,14}\right]$ cations in all studied $\mathrm{IL} /$ water mixtures as a function of water mole fractions: (a) $\mathrm{C}_{\text {hexyl }}-\mathrm{C}_{\text {hexyl }}$; (b) $\mathrm{C}_{\text {tetradecyl }}-$ $\mathrm{C}_{\text {tetradecyl. }}$.

mixtures with a low water concentration, the added water molecules have little effect on the spatial correlations of these terminal methyl groups due to the fact that most of the added water molecules are mainly embedded in cavities between neighboring ionic species, and are far away from the terminal carbon atoms in the four hydrophobic alkyl chains. That is, the favorable hydrogen bonded interactions and the strong electrostatic interactions between the central polar segments in $\left[\mathrm{P}_{6,6,6,14}\right]$ cations and water molecules dominate the relative distribution of water molecules in these $\mathrm{IL} /$ water mixtures. The spatial correlations between terminal carbon atoms, either in the hexyl or in the tetradecyl chains, are considerably enhanced in IL/water mixtures with water mole fractions of $x_{\text {water }}>0.80$, in which large water clusters are explicitly formed, as clearly illustrated in representative snapshots in Figure 2. It is the hydrophobic nature of terminal methyl groups, as well as other methylene groups consisting of $\left[\mathrm{P}_{6,6,6,14}\right]$ cations, that drive their aggregation in water concentrated mixtures.

The competitive interactions between $\left[\mathrm{P}_{6,6,6,14}\right]$ cations and water molecules are further verified by analyzing the intermolecular interaction energies between these two species. Figure 12 presents the short-range dispersion energies and the long-range electrostatic energies, as well as the total 


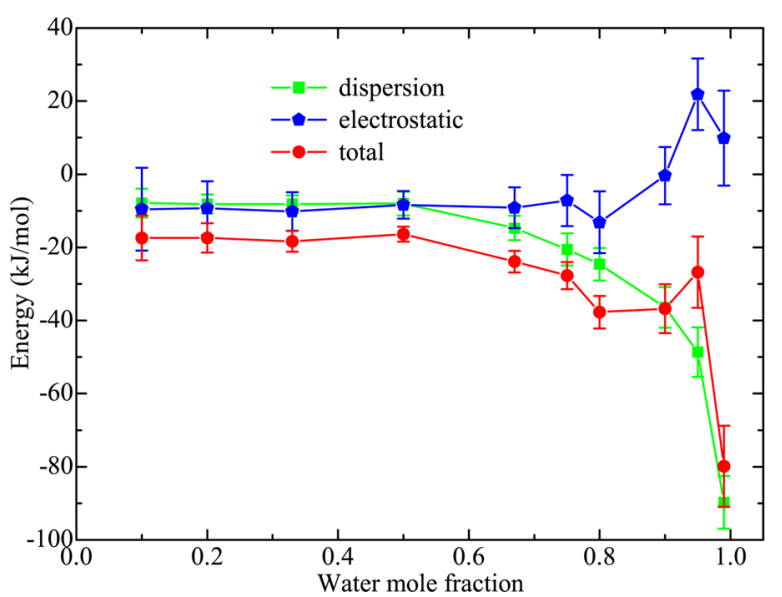

Figure 12. Calculated short-range dispersion energies and long-range electrostatic energies, as well as the total intermolecular interaction energies between $\left[\mathrm{P}_{6,6,6,14}\right]$ cations and water molecules in all studied $\mathrm{IL} /$ water mixtures as a function of water mole fractions.

intermolecular interaction energies between $\left[\mathrm{P}_{6,6,6,14}\right]$ cations and water molecules in the $\mathrm{IL} /$ water mixtures with varied water mole fractions. The short-ranged dispersion energies are comparable with the corresponding long-ranged electrostatic energies in IL/water mixtures with water mole fractions of $x_{\text {water }}$ $\leq 0.50$ due to the dispersed water monomers in cavities between neighboring ionic species. With an increase in the water concentration, a decrease in dispersion energies and an increase in electrostatic energies between these two species indicate the enhanced hydrophobic tendencies of alkyl chains in $\left[\mathrm{P}_{6,6,6,14}\right]$ cations and the aggregation of additional water molecules to expand isolated polar domains before the formation of an interpenetrating polar network. In IL/water mixtures with water mole fractions of $x_{\text {water }}>0.95$, both dispersion and electrostatic energies decrease, as well as the total intermolecular interaction energies, indicating the formation of bicontinuous networks and micelle-like aggregates scattered in a branched water network.

Concerning the evolution of the microscopic liquid structure and the transition of the local ionic organization, all studied IL/ water mixtures can be divided into four distinct structural regimes in the whole water concentration range.

- Neat IL and IL/water mixtures with water mole fractions of $0.0<x_{\text {water }}<0.50$. In the neat IL sample, $\left[\mathrm{P}_{6,6,6,14}\right]$ cations and $[\mathrm{BOB}]$ anions are closely coupled together via strong electrostatic interactions. The microscopic liquid structure is characterized by a connected apolar network consisting of the relatively large and volumeoccupying alkyl substituents in $\left[\mathrm{P}_{6,6,6,14}\right]$ cations and the isolated polar domains consisting of the central segments in $\left[\mathrm{P}_{6,6,6,14}\right]$ cations and $[\mathrm{BOB}]$ anions. The local ionic organization is described by direct contact ion pairs. In IL/water mixtures with a low water content, most of the added water molecules are dispersed in cavities between neighboring ionic species; that is, each $\left[\mathrm{P}_{6,6,6,14}\right][\mathrm{BOB}]$ ion pair shares one common water molecule, and the corresponding local ionic environment is characterized by solvent-shared ion pairs through cation-water-anion triple complexes. Furthermore, water dimers, trimers, and even small water clusters are formed in these IL/ water mixtures as the water concentration increases.
- IL/water mixtures with intermediate water mole fractions of $0.50<x_{\text {water }}<0.80$. Large water clusters appear to dominate the size distribution due to the fact that there is not a sufficient void space to accommodate an increasing number of water molecules. A distinct water aggregation state emerges, that is, the chain-like water structures characterized by anion $\cdots\left(\mathrm{H}_{2} \mathrm{O}\right)_{n} \cdots$ anion multiple complexes. With a gradual increase in the water concentration, these chain-like water structures grow and serve as bridges to connect more ionic species, as well as to mediate their relative distribution and orientation in IL/ water mixtures. Hence, the local ionic organization of these IL/water mixtures is characterized by solventmediated ion pairs through multiple complexes, leading to the enhanced spatial correlations between ionic species.

- IL/water mixtures with water mole fractions of $0.80<$ $x_{\text {water }}<0.95$. In these water concentrated mixtures, the branched sort of connectivity allows water molecules to dynamically percolate through the entire simulation cell and form a three-dimensional water network, leading to the microscopic liquid structure described by interpenetrating polar and apolar networks. Even though there are many additional water molecules present in these mixtures, the local ionic environment is not disrupted; that is, the central polar segments of $\left[\mathrm{P}_{6,6,6,14}\right]$ cations and $[\mathrm{BOB}]$ anions remain in close proximity to each other through sharing one or more water molecules. This is attributed to the strong electrostatic interactions and the favorable hydrogen bonded interactions between ionic species.

- IL/water mixtures with water mole fractions of $x_{\text {water }}>$ 0.95. Further progressive dilution of IL/water mixtures leads to the percolation limit of IL in water; i.e., upon further dilution, the connected apolar network consisting of the hydrophobic alkyl chains in $\left[\mathrm{P}_{6,6,6,14}\right]$ cations (already stretched to its limit) starts to break up into loose micelle-like aggregates and loses its continuous nature. In extremely diluted mixtures, such as with a water mole fraction of $x_{\text {water }}=0.99$ (data not shown), a self-organized micelle-like structure characterized by a hydrophobic core and a hydrophilic shell is formed and isolated in a highly branched water network.

The striking microscopic liquid structure and the distinct local ionic environment in IL/water mixtures are attributed to the competition between favorable hydrogen bonded interactions and strong electrostatic interactions between the central polar segments in ionic species, and the dispersion interactions between the hydrophobic alkyl chains in $\left[\mathrm{P}_{6,66,6,14}\right]$ cations. Compared with the protic imidazolium-based ILs extensively studied in previous works, ${ }^{37,38,50,51,53-55,59}$ the tetraalkylphosphonium cations exhibit distinct features. They are much more hydrophobic, asymmetric, and bulky, making it more difficult for both water molecules and anionic groups to approach the positively charged center of the tetraalkylphosphonium cations. In contrast, the imidazolium-based cations have only one alkyl chain hindering the access of other molecules to the positively charged imidazolium rings. Concerning the particular microstructural analyses in the present work, the evolution of the microscopic liquid structure and the local ionic environment of studied $\left[\mathrm{P}_{6,6,6,14}\right][\mathrm{BOB}] \mathrm{IL} /$ water mixtures with different water mole fractions is generally similar to that of aqueous solutions 
of imidazolium-based ILs. ${ }^{37,38,50,51,53-55,59}$ More specifically, the evolution of the microscopic liquid structure and the local ionic environment, either for currently studied $\left[\mathrm{P}_{6,6,6,14}\right][\mathrm{BOB}]$ $\mathrm{IL} /$ water mixtures or for previously investigated 1-octyl-3methylimidazolium nitrate ([OMIM $]\left[\mathrm{NO}_{3}\right]$ ) IL/water mixtures, ${ }^{50}$ are characterized by contact ion pairs, solvent-mediated ion pairs, interpenetrating polar and apolar networks, and eventually loose micelle-like aggregates in a highly branched water network as water concentration increases. In addition to the distinct changes in local ionic environment between water molecules, polar anions, and polar segments in cations, there are also considerably enhanced spatial correlations between terminal carbon atoms in respective cations, which promote their prominent aggregation in water concentrated mixtures. From a structural perspective, this microstructural evolution might be a general feature of the $\left[\mathrm{P}_{6,6,6,14}\right][\mathrm{BOB}] \mathrm{IL} /$ water mixtures and the imidazolium-based IL/water mixtures, attributing to the amphiphilic surfactant-like behavior of tetraalkylphosphonium and imidazolium-based cations in aqueous solutions, and the competition between favorable interactions between polar segments in ionic species, and persistent hydrophobic interactions between alkyl chains in respective cations. Such an evolution of the microscopic liquid structure and a heterogeneous local ionic environment in the currently studied $\left[\mathrm{P}_{6,6,6,14}\right][\mathrm{BOB}] \mathrm{IL} /$ water mixtures contribute directly to striking thermodynamic and particular transport properties of these IL/water mixtures, which will be systematically discussed in our following work.

\section{CONCLUSIONS}

In the present work, atomistic molecular dynamics simulations were performed to study the evolution of the microscopic liquid structure and the local ionic organization of the $\left[\mathrm{P}_{6,6,6,14}\right][\mathrm{BOB}] \mathrm{IL} /$ water mixtures as a function of the water concentration. Detailed analyses of radial and spatial distribution functions, nearest neighbor statistics, hydrogen bond network, as well as water clustering analysis indicate that the local microscopic ionic environment is distinct and characterized by heterogeneous ionic structures.

Four distinct structural regimes are identified within the full water concentration range. With a gradual increase in the water concentration, the local ionic structure is characterized by direct contact ion pairs and solvent-shared ion pairs through cationwater-anion triple complexes $\left(0.00 \leq x_{\text {water }}<0.50\right)$, solventmediated ion pairs through multiple complexes $\left(0.50<x_{\text {water }}<\right.$ $0.80)$, interpenetrating polar and apolar networks $\left(0.80<x_{\text {water }}\right.$ $<0.95$ ), and eventually loose micelle-like aggregates in a highly branched water network $\left(x_{\text {water }}>0.95\right)$. The striking structural evolution in the $\left[\mathrm{P}_{6,6,6,14}\right][\mathrm{BOB}] \mathrm{IL} /$ water mixtures is attributed to the competition between favorable hydrogen bonded interactions and strong electrostatic interactions between the central polar segments in ionic species and the dispersion interactions between the hydrophobic alkyl chains in $\left[\mathrm{P}_{6,6,6,14}\right]$ cations.

\section{AUTHOR INFORMATION}

\section{Corresponding Authors}

*Phone: 00468 162372. Fax: 00468 152187. E-mail: yonglei. wang@mmk.su.se.

*Phone: 00468 7909549. Fax: 00468 152187. E-mail: aatto. laaksonen@mmk.su.se.

\section{Notes}

The authors declare no competing financial interest.

\section{ACKNOWLEDGMENTS}

We gratefully acknowledge the financial support from the Knut and Alice Wallenberg Foundation (Project number KAW 2012.0078) and the Swedish Research Council (Project numbers 2013-5171, 2013-4725, and 2014-4694). All computer simulations were performed using computational resources provided by the Swedish National Infrastructure for Computing (SNIC) at PDC, HPC2N, and NSC. We also thank Prof. Lars Kloo (KTH) and Dr. Faiz Ullah Shah (LTU) for their fruitful discussion and constructive comments on this paper.

\section{REFERENCES}

(1) Greaves, T. L.; Drummond, C. J. Protic Ionic Liquids: Properties and Applications. Chem. Rev. 2008, 108, 206-237.

(2) Niedermeyer, H.; Hallett, J. P.; Villar-Garcia, I. J.; Hunt, P. A.; Welton, T. Mixtures of Ionic Liquids. Chem. Soc. Rev. 2012, 41, 77807802.

(3) Weingärtner, H. Understanding Ionic Liquids at the Molecular Level: Facts, Problems, and Controversies. Angew. Chem., Int. Ed. 2008, 47, 654-670.

(4) Plechkova, N. V.; Seddon, K. R. Applications of Ionic Liquids in the Chemical Industry. Chem. Soc. Rev. 2008, 37, 123-150.

(5) Hallett, J. P.; Welton, T. Room-Temperature Ionic Liquids: Solvents for Synthesis and Catalysis. 2. Chem. Rev. 2011, 111, 35083576.

(6) Wasserscheid, P.; Keim, W. Ionic Liquids-New "Solutions" for Transition Metal Catalysis. Angew. Chem., Int. Ed. 2000, 39, 37723789.

(7) Ma, Z.; Yu, J.; Dai, S. Preparation of Inorganic Materials Using Ionic Liquids. Adv. Mater. 2010, 22, 261-285.

(8) Yu, B.; Liu, Z.; Ma, C.; Sun, J.; Liu, W.; Zhou, F. Ionic Liquid Modified Multi-Walled Carbon Nanotubes as Lubricant Additive. Tribol. Int. 2015, 81, 38-42.

(9) Zhou, F.; Liang, Y.; Liu, W. Ionic Liquid Lubricants: Designed Chemistry for Engineering Applications. Chem. Soc. Rev. 2009, 38, 2590-2599.

(10) Liu, J.; Li, J.; Yu, B.; Ma, B.; Zhu, Y.; Song, X.; Cao, X.; Yang, W.; Zhou, F. Tribological Properties of Self-Assembled Monolayers of Catecholic Imidazolium and the Spin-Coated Films of Ionic Liquids. Langmuir 2011, 27, 11324-11331.

(11) Shah, F. U.; Glavatskih, S.; MacFarlane, D. R.; Somers, A.; Forsyth, M.; Antzutkin, O. N. Novel Halogen-Free Chelated Orthoborate-Phosphonium Ionic Liquids: Synthesis and Tribophysical Properties. Phys. Chem. Chem. Phys. 2011, 13, 12865-12873.

(12) Somers, A. E.; Howlett, P. C.; MacFarlane, D. R.; Forsyth, M. A Review of Ionic Liquid Lubricants. Lubricants 2013, 1, 3-21.

(13) Castejón, H. J.; Wynn, T. J.; Marcin, Z. M. Wetting and Tribological Properties of Ionic Liquids. J. Phys. Chem. B 2014, 118, 3661-3668.

(14) Taher, M.; Shah, F. U.; Filippov, A.; de Baets, P.; Glavatskih, S.; Antzutkin, O. N. Halogen-Free Pyrrolidinium Bis(mandelato)borate Ionic Liquids: Some Physicochemical Properties and Lubrication Performance as Additives to Polyethylene Glycol. RSC Adv. 2014, 4, 30617-30623.

(15) Han, X.; Armstrong, D. W. Ionic Liquids in Separations. Acc. Chem. Res. 2007, 40, 1079-1086.

(16) Myers, C.; Pennline, H.; Luebke, D.; Ilconich, J.; Dixon, J. K.; Maginn, E. J.; Brennecke, J. F. High Temperature Separation of Carbon Dioxide/Hydrogen Mixtures Using Facilitated Supported Ionic Liquid Membranes. J. Membr. Sci. 2008, 322, 28-31.

(17) Swatloski, R. P.; Spear, S. K.; Holbrey, J. D.; Rogers, R. D. Dissolution of Cellose with Ionic Liquids. J. Am. Chem. Soc. 2002, 124, 4974-4975. 
(18) Brandt, A.; Ray, M. J.; To, T. Q.; Leak, D. J.; Murphy, R. J.; Welton, T. Ionic Liquid Pretreatment of Lignocellulosic Biomass with Ionic Liquid-Water Mixtures. Green Chem. 2011, 13, 2489-2499.

(19) Passos, H.; Freire, M. G.; Coutinho, J. A. P. Ionic Liquid Solutions as Extractive Solvents for Value-added Compounds From Biomass. Green Chem. 2014, 16, 4786-4815.

(20) Hough, W. L.; Smiglak, M.; Rodríguez, H.; Swatloski, R. P.; Spear, S. K.; Daly, D. T.; Pernak, J.; Grisel, J. E.; Carliss, R. D.; Soutullo, M. D.; et al. The Third Evolution of Ionic Liquids: Active Pharmaceutical Ingredients. New J. Chem. 2007, 31, 1429-1436.

(21) Gardas, R. L.; Costa, H. F.; Freire, M. G.; Carvalho, P. J.; Marrucho, I. M.; Fonseca, I. M. A.; Ferreira, A. G. M.; Coutinho, J. A. P. Densities and Derived Thermodynamic Properties of Imidazolium-, Pyridinium-, Pyrrolidinium-, and Piperidinium-Based Ionic Liquids. J. Chem. Eng. Data 2008, 53, 805-811.

(22) Sánchez, L. G.; Espel, J. R.; Onink, F.; Meindersma, G. W.; de Haan, A. B. Density, Viscosity, and Surface Tension of Synthesis Grade Imidazolium, Pyridinium, and Pyrrolidinium Based Room Temperature Ionic Liquids. J. Chem. Eng. Data 2009, 54, 2803-2812.

(23) Stegemann, H.; Rohde, A.; Reiche, A.; Schnittke, A. Füllbier, H. Room Temperature Molten Polyiodides. Electrochim. Acta 1992, 37, 379-383.

(24) Gerhard, D.; Alpaslan, S. C.; Gores, H. J.; Uerdingen, M.; Wasserscheid, P. Trialkylsulfonium Dicyanamides-A New Family of Ionic Liquids with Very Low Viscosities. Chem. Commun. 2005, 50805082.

(25) Zhang, Q.; Liu, S.; Li, Z.; Li, J.; Chen, Z.; Wang, R.; Lu, L.; Deng, Y. Novel Cyclic Sulfonium-Based Ionic Liquids: Synthesis Characterization, and Physicochemical Properties. Chem.-Eur. J. 2009, 15, 765-778.

(26) Fraser, K. J.; MacFarlane, D. R. Phosphonium-Based Ionic Liquids: An Overview. Aust. J. Chem. 2009, 62, 309-321.

(27) Del Sesto, R. E.; Corley, C.; Robertson, A.; Wilkes, J. S. Tetraalkylphosphonium-Based Ionic Liquids. J. Organomet. Chem. 2005, 690, 2536-2542.

(28) Kagimoto, J.; Fukumoto, K.; Ohno, H. Effect of Tetrabutylphosphonium Cation on the Physicochemical Properties of AminoAcid Ionic Liquids. Chem. Commun. 2006, 2254-2256.

(29) Yu, B.; Bansal, D. G.; Qu, J.; Sun, X.; Luo, H.; Dai, S.; Blau, P. J.; Bunting, B. G.; Mordukhovich, G.; Smolenski, D. J. Oil-miscible and Non-corrosive Phosphonium-Based Ionic Liquids as Candidate Lubricant Additives. Wear 2012, 289, 58-64.

(30) Shah, F. U.; Glavatskih, S.; Antzutkin, O. N. Boron in Tribology: From Borates to Ionic Liquids. Tribol. Lett. 2013, 51, 281-301.

(31) Atefi, F.; Garcia, M. T.; Singer, R. D.; Scammells, P. J. Phosphonium Ionic Liquids: Design, Synthesis and Evaluation of Biodegradability. Green Chem. 2009, 11, 1595-1604.

(32) Minami, I. Ionic Liquids in Tribology. Molecules 2009, 14, 2286-2305.

(33) Palacio, M.; Bhushan, B. A Review of Ionic Liquids for Green Molecular Lubrication in Nanotechnology. Tribol. Lett. 2010, 40, 247268.

(34) Petkovic, M.; Seddon, K. R.; Rebelo, L. P. N.; Pereira, C. S. Ionic Liquids: A Pathway to Environmental Acceptability. Chem. Soc. Rev. 2011, 40, 1383-1403.

(35) Nockemann, P.; Binnemans, K.; Driesen, K. Purification of Imidazolium Ionic Liquids for Spectroscopic Applications. Chem. Phys. Lett. 2005, 415, 131-136.

(36) Stark, A.; Behrend, P.; Braun, O.; Müller, A.; Ranke, J.; Ondruschka, B.; Jastorff, B. Purity Specification Methods for Ionic Liquids. Green Chem. 2008, 10, 1152-1161.

(37) Cammarata, L.; Kazarian, S. G.; Salter, P. A.; Welton, T. Molecular States of Water in Room Temperature Ionic Liquids. Phys. Chem. Chem. Phys. 2001, 3, 5192-5200.

(38) Schröder, U.; Wadhawan, J. D.; Compton, R. G.; Marken, F.; Suarez, Z.; Consorti, C. S.; de Souza, R. F.; Dupont, J. Water-Induced Accelerated Ion Diffusion: Voltammetric Studies in 1-methyl-3-[2,6(S)-dimethylocten-2-yl] Imidazolium Tetrafluoroborate, 1-butyl-3- methylimidazolium Tetrafluoroborate and Hexafluorophosphate Ionic Liquids. New J. Chem. 2000, 24, 1009-1015.

(39) Endres, F.; El Abedin, S. Z. Air and Water Stable Ionic Liquids in Physical Chemistry. Phys. Chem. Chem. Phys. 2006, 8, 2101-2116. (40) Wang, Y.-L.; Shah, F. U.; Glavatskih, S.; Antzutkin, O. N.; Laaksonen, A. Atomistic Insight into Orthoborate-Based Ionic Liquids: Force Field Development and Evaluation. J. Phys. Chem. B 2014, 118, $8711-8723$

(41) Berendsen, H. J. C.; Grigera, J. R.; Straatsma, T. P. The Missing Term in Effective Pair Potentials. J. Phys. Chem. 1987, 91, 6269-6271.

(42) Lyubartsev, A. P.; Laaksonen, A. M.DynaMix-A Scalable Portable Parallel MD Simulation Package for Arbitrary Molecular Mixtures. Comput. Phys. Commun. 2000, 128, 565-589.

(43) Tuckerman, M.; Berne, B. J.; Martyna, G. J. Reversible Multiple Time Scale Molecular Dynamics. J. Chem. Phys. 1992, 97, 1990.

(44) Wang, Y.; Voth, G. A. Unique Spatial Heterogeneity in Ionic Liquids. J. Am. Chem. Soc. 2005, 127, 12192-12193.

(45) Canongia Lopes, J. N.; Pádua, A. A. Nanostructural Organization in Ionic Liquids. J. Phys. Chem. B 2006, 110, 3330-3335.

(46) Wang, Y.-L.; Lyubartsev, A.; Lu, Z.-Y.; Laaksonen, A. Multiscale Coarse-Grained Simulations of Ionic Liquids: Comparison of Three Approaches to Derive Effective Potentials. Phys. Chem. Chem. Phys. 2013, 15, 7701-7712.

(47) Wang, Y.-L.; Laaksonen, A.; Lu, Z.-Y. Influence of Ionic Liquid Film Thickness on Ion Pair Distributions and Orientations at Graphene and Vacuum Interfaces. Phys. Chem. Chem. Phys. 2013, 15, 13559-13569.

(48) Wang, Y.-L.; Laaksonen, A. Interfacial Structure and Orientation of Confined Ionic Liquids on Charged Quartz Surfaces. Phys. Chem. Chem. Phys. 2014, 16, 23329-23339.

(49) The extremely diluted $\left[\mathrm{P}_{6,6,14}\right][\mathrm{BOB}] \mathrm{IL} /$ water mixture with a water mole fraction of $x_{\text {water }}=0.99$ consists of $32\left[\mathrm{P}_{6,6,6,14}\right][\mathrm{BOB}]$ ion pairs and 3168 water molecules, and the corresponding water mass fraction is $\mathrm{wt}_{\text {water }}=0.7267$.

(50) Jiang, W.; Wang, Y.; Voth, G. A. Molecular Dynamics Simulation of Nanostructural Organization in Ionic Liquid/Water Mixtures. J. Phys. Chem. B 2007, 111, 4812-4818.

(51) Feng, S.; Voth, G. A. Molecular Dynamics Simulations of Imidazolium-Based Ionic Liquid/Water Mixtures: Alkyl Side Chain Length and Anion Effects. Fluid Phase Equilib. 2010, 294, 148-156.

(52) Hayes, R.; Imberti, S.; Warr, G. G.; Atkin, R. How Water Dissolves in Protic Ionic Liquids. Angew. Chem., Int. Ed. 2012, 51, $7468-7471$.

(53) Spickermann, C.; Thar, J.; Lehmann, S. B. C.; Zahn, S.; Hunger, J.; Buchner, R.; Hunt, P. A.; Welton, T.; Kirchner, B. Why Are Ionic Liquid Ions Mainly Associated in Water? A Car-Parrinello Study of 1Ethyl-3-methyl-imidazolium Chloride Water Mixture. J. Chem. Phys. 2008, 129, 104505.

(54) Zahn, S.; Wendler, K.; Delle Site, L.; Kirchner, B. Depolarization of Water in Protic Ionic Liquids. Phys. Chem. Chem. Phys. 2011, 13, 15083-15093.

(55) Zhong, X.; Fan, Z.; Liu, Z.; Cao, D. Local Structure Evolution and Its Connection to Thermodynamic and Transport Properties of 1butyl-3-methylimidazolium Tetrafluoroborate and Water Mixtures by Molecular Dynamics Simulations. J. Phys. Chem. B 2012, 116, 32493263.

(56) Usula, M.; Mocci, F.; Marincola, F. C.; Porcedda, S.; Gontrani, L.; Caminiti, R. The Structural Organization of N-methyl-2pyrrolidone+Water Mixtures: A Densitometry, X-ray Diffraction, and Molecular Dynamics Study. J. Chem. Phys. 2014, 140, 124503.

(57) Laaksonen, L. A. Graphics Program for the Analysis and Display of Molecular Dynamics Trajectories. J. Mol. Graphics 1992, 10, 33-34.

(58) Bergman, D. L.; Laaksonen, L.; Laaksonen, A. Visualization of Solvation Structures in Liquid Mixtures. J. Mol. Graphics Modell. 1997, $15,301-306$

(59) Hanke, C.; Lynden-Bell, R. A Simulation Study of WaterDialkylimidazolium Ionic Liquid Mixtures. J. Phys. Chem. B 2003, 107, 10873-10878. 
(60) Hess, B.; Kutzner, C.; van der Spoel, D.; Lindahl, E. GROMACS 4: Algorithms for Highly Efficient, Load-balanced, and Scalable Molecular Simulation. J. Chem. Theory Comput. 2008, 4, 435-447.

(61) Lyubartsev, A.; Laaksonen, A. Determination of Effective Pair Potentials From Ab Initio Simulations: Application to Liquid Water. Chem. Phys. Lett. 2000, 325, 15-21. 